\title{
The amplitude of fNIRS hemodynamic response in the visual cortex unmasks autistic traits in typically developing children
}

\author{
Raffaele Mazziotti ${ }^{1,5}$, Elena Scaffei ${ }^{1,2,5}$, Eugenia Conti ${ }^{1}$, Viviana Marchi ${ }^{1}$, Riccardo Rizzi $\mathbb{D}^{1,2}$, Giovanni Cioni ${ }^{1,3}$, Roberta Battini ${ }^{1,3}$ and \\ Laura Baroncelli $\mathbb{D}^{1,4}$
}

(c) The Author(s) 2022

\begin{abstract}
Autistic traits represent a continuum dimension across the population, with autism spectrum disorder (ASD) being the extreme end of the distribution. Accumulating evidence shows that neuroanatomical and neurofunctional profiles described in relatives of ASD individuals reflect an intermediate neurobiological pattern between the clinical population and healthy controls. This suggests that quantitative measures detecting autistic traits in the general population represent potential candidates for the development of biomarkers identifying early pathophysiological processes associated with ASD. Functional near-infrared spectroscopy (fNIRS) has been extensively employed to investigate neural development and function. In contrast, the potential of fNIRS to define reliable biomarkers of brain activity has been barely explored. Features of non-invasiveness, portability, ease of administration, and lowoperating costs make fNIRS a suitable instrument to assess brain function for differential diagnosis, follow-up, analysis of treatment outcomes, and personalized medicine in several neurological conditions. Here, we introduce a novel standardized procedure with high entertaining value to measure hemodynamic responses (HDR) in the occipital cortex of adult subjects and children. We found that the variability of evoked HDR correlates with the autistic traits of children, assessed by the Autism-Spectrum Quotient. Interestingly, HDR amplitude was especially linked to social and communication features, representing the core symptoms of ASD. These findings establish a quick and easy strategy for measuring visually-evoked cortical activity with fNIRS that optimize the compliance of young subjects, setting the background for testing the diagnostic value of fNIRS visual measurements in the ASD clinical population.
\end{abstract}

Translational Psychiatry (2022)12:53; https://doi.org/10.1038/s41398-022-01820-5

\section{INTRODUCTION}

Autism spectrum disorder (ASD) is a heterogeneous developmental condition that involves persistent challenges in social interactions, restricted/repetitive behaviors, and the lack of behavioral and cognitive flexibility [1]. Since the pioneering work by Lorna Wing [2], increasing epidemiological evidence indicates that autistic traits are continuously distributed across the general population [3, 4]. This is due to the complex genetic and epigenetic inheritance pattern of ASD, where multiple candidate loci contribute to the pathogenesis of the disease $[5,6]$. Milder autistic traits have been termed the extended or broader autism phenotype (BAP), with BAP features being particularly prevalent in first- and second-degree relatives of individuals with ASD [6-9]. Much research is currently focused on siblings of children diagnosed with ASD, namely high-risk infants (HR), because around $20 \%$ of them receive a diagnosis of ASD within the third year of life [10] and a further 20-30\% develop neurodevelopmental conditions [11]. Moreover, a high rate of BAP symptoms has been documented in this population [12]. The prospective study of HR children allows to detect behavioral risk signs or biomarkers of neurodevelopmental disorders at a very early age, before the full-blown clinical expression, while the investigation of
BAP features in the general population might be helpful to dissect clinical subtypes and set-up personalized intervention strategies according to developmental stages.

Over the last decade, the biological dimension of ASD has been largely explored, thanks to the growing availability of advanced tools to explore brain correlates of neurological disorders, including high-density EEG, magnetoencephalography, positron emission tomography, magnetic resonance imaging (MRI), and functional near-infrared spectroscopy (fNIRS) $[8,13,14]$. A number of studies reported defective neuroanatomical and neurofunctional features in individuals with ASD, suggesting that a dysfunction of specific brain areas might underlie the core symptoms of ASD [15-19]. Interestingly, relatives of autistic probands, even when not behaviorally impaired, display neurostructural and neurofunctional patterns significantly different from healthy controls and correlated to BAP features [8]. Since ASD and broader autistic manifestations share common genetic variants and neurobiological susceptibility factor [20], the general population emerges as a suitable testing bed for the development of quantitative measures detecting hallmarks of autism.

FNIRS is an optical imaging technique that allows quantifying oxygen consumption in different regions of the cerebral cortex,

\footnotetext{
${ }^{1}$ Department of Developmental Neuroscience, IRCCS Stella Maris Foundation, I-56128 Pisa, Italy. ${ }^{2}$ Department of Neuroscience, Psychology, Drug Research and Child Health NEUROFARBA, University of Florence, I-50135 Florence, Italy. ${ }^{3}$ Department of Clinical and Experimental Medicine, University of Pisa, Pisa, Italy. ${ }^{4}$ Institute of Neuroscience, National Research Council (CNR), I-56124 Pisa, Italy. ${ }^{5}$ These authors contributed equally: Raffaele Mazziotti, Elena Scaffei. ${ }^{凶}$ email: baroncelli@in.cnr.it
}

Received: 3 August 2021 Revised: 17 January 2022 Accepted: 19 January 2022

Published online: 08 February 2022 
providing an indirect measure of neuronal activity [21, 22]. This blood-oxygen-level-dependent (BOLD) signal is similar to that detected with functional MRI (fMRI) [23]. However, fNIRS is more tolerant to motion artifacts than $\mathrm{fMRl}$, and the development of robust methods for motion detection and correction allowed to avoid sedation in children $[24,25]$. Furthermore, fNIRS has the advantage of being totally non-invasive, low-cost, portable, noiseless, and endowed with high experimental flexibility and no setting constraints. This methodological strength provides the fNIRS with a high ecological value for investigating neural circuit maturation either in typically developing children or clinically relevant populations $[24,26]$.

Although the use of fNIRS in autism research is still an emerging area, a number of studies aiming to decipher the neuronal mechanisms and circuits underlying ASD evaluated different aspects of brain function and organization, including resting-state and task-evoked responses $[27,28]$. Coherence analyses of restingstate hemodynamic activity showed weaker local and interhemispheric functional connectivity in different cortical regions [29-34]. Moreover, individuals on the autism spectrum present patterns of atypical activity, including reduced hemodynamic responses within specific brain regions, bilateral differences in neuronal activation, and the lack of cortical specialization, in tasks ranging from sensory perception [35] to executive functions [36], social perception [37-40], joint attention [41-43], imitation [44, 45], facial and emotional processing [46-49], speech perception and language [50-53]. The majority of studies targeting evoked brain activity were focused on the prefrontal and the temporal cortex [28], where symptom severity seems to be inversely correlated with the degree of cortical activation $[45,46]$.

Growing evidence suggests that fNIRS might be a candidate biomarker for several neuropsychiatric disorders, including ASD [32, 54-58]. In particular, functional network efficiency [32], weighted separability of NIRS signals [59], multi-layer neural networks and sample entropy of spontaneous hemodynamic fluctuations $[57,60]$ have been proposed as auxiliary diagnosis indexes for ASD. However, all these approaches require complex algorithms to extract high-level features from the fNIRS raw data, while fitness, applicability, and translational value of biomarkers greatly depend on their ease of use. In this framework, the analysis of visual phenotype has become an important model to evaluate cortical processing in different neurodevelopmental conditions [61-66]. Indeed, electrophysiological measurement of visually evoked responses has been introduced as a quantitative method to assess brain function in Rett syndrome [61, 67], and hemodynamic responses (HDR) emerged as a potential longitudinal biomarker for CDKL5 Deficiency Disorder and Creatine Transporter Deficiency in murine models $[65,66]$.

Since clinical studies suggested a dysregulation of sensory processing and functional connectivity in the visual cortex of ASD subjects [68-70], and atypical visual processing has been implicated in the neurobiology of autism [71, 72], we hypothesized that fNIRS visual measures might represent a tool to quantitively assess inter-individual differences in autistic traits. The stimulation protocol used in most fNIRS and electrophysiological studies quantitively assessing visual responses in both typical and clinical populations consisted of reversing black and white checkerboard patterns spaced by baseline intervals with a grey isoluminant screen $[67,71-75]$. The same paradigm has been also employed to measure visually evoked potentials in autistic individuals [71, 72]. The entertaining value of the checkerboard/ gray alternation, however, is quite low, possibly reducing the experimental compliance of children with intellectual and neurodevelopmental disorders.

Thus, this work had three specific aims: (1) to set up a novel standardized procedure to assess HDR in the occipital cortex; (2) to test the feasibility and reliability of fNIRS measurements in typical adults and children using this innovative stimulation protocol; (3) to investigate the correlation between HDR and broad autism dimensions, evaluated with the Autism-Spectrum Quotient (AQ $[76,77])$, in the general population.

\section{RESULTS}

An animated cartoon-based stimulus is able to evoke visual responses in the adult cortex

We measured the cortical HDR function [78] elicited by a reversing checkerboard pattern in the adult population. In agreement with the previous literature $[69,79]$, we obtained a significant activation of the occipital cortex in response to different conditions of visual stimulation (Fig. 1 and Fig. S1). Grand averages across adult participants (see Table 1 for demographics) of Total $\mathrm{Hb}(\mathrm{THb})$, $\mathrm{OxyHb}(\mathrm{OHb})$ and DeoxyHb $(\mathrm{DHb})$ concentration changes are plotted in Fig. 2. Using a classic mean-luminance gray screen as baseline, statistical analysis revealed a significant main effect of the checkerboard stimulus (S) with respect to the blank presentation for all HDR metrics (Radial Stimulus condition, RS, Fig. 2A; see Table S1 for statistical details).

To increase the entertaining quality of our experimental paradigm, we devised an innovative visual stimulation protocol blending the checkerboard pattern with an isoluminant commercial cartoon, thus serving as a reference baseline (Fig. 1 and Fig. $\mathrm{S} 1)$. We found a significant increase of $\mathrm{THb}$ and $\mathrm{OHb}$, with a parallel reduction of $\mathrm{DHb}$ concentration, in response to $\mathrm{S}$ appearance, reflecting the functional activation of visual areas in this condition as well. The cortical response was independent from the cartoon employed as baseline: a comparable HDR, indeed, was clearly elicited both when the baseline movie was fixed a priori by the experimenter (Cartoon Fixed condition, CF; Fig. 2B, Table S1) and when the cartoon was freely selected by the tested subject (Cartoon Chosen condition, CC; Fig. 2C, Table S1). Interestingly, a significant pattern of correlations emerged among HDR metrics recorded with different stimulating conditions (Fig. S2; see Table S1 for statistical details), indicating that the quality of visual input does not quantitatively impact HDR. The amplitude of cortical activation was only slightly smaller in response to CF and CC, with the range of $\mathrm{OHb}$ and $\mathrm{DHb}$ fluctuations being significantly lower with respect to that evoked by RS (Fig. 2D; Table S1).

Within the CF condition, we also established that the baseline cartoon does not affect the degree of visual activation: indeed, a comparable modification of $\mathrm{THb}, \mathrm{OHb}$, and $\mathrm{DHb}$ concentrations was recorded using "The Lion King", "The Powerpuff Girls", "Peppa Pig" or "Kung Fu Panda" (Fig. S3A; Table S1). Furthermore, no differences of visually evoked responses were detected modulating the contrast level of the baseline cartoon: $\mathrm{THb}, \mathrm{OHb}$, and $\mathrm{DHb}$ fluctuations, indeed, were comparable when a fixed baseline cartoon was presented at 20,40, or $80 \%$ of contrast (Fig. 2E; Fig. S3B-D; Table S1). Finally, the response latency was homogenous in RS, CF, and CC conditions (Fig. S4A; Table S1).

Altogether, these results demonstrate the validity of this innovative stimulation procedure to evoke a significant and reliable response in the occipital cortex preserving inter-subject variability.

\section{The cartoon paradigm was reliable in eliciting cortical responses in children}

We measured cortical responses in typically developing children (see Table 2 for demographics) viewing the radial checkerboard blended with the animated cartoon. We compared three different conditions: each subject, indeed, was asked to select two cartoons of their preference for the baseline, and the first choice was employed for the low-contrast (cartoon 1 low contrast, 20\%, L1) and the high-contrast (cartoon 1 high contrast, 80\%, $\mathrm{H} 1$ ) stimulation, while the second cartoon was presented only at low-contrast (cartoon 2 low contrast, L2; Fig. S1). 


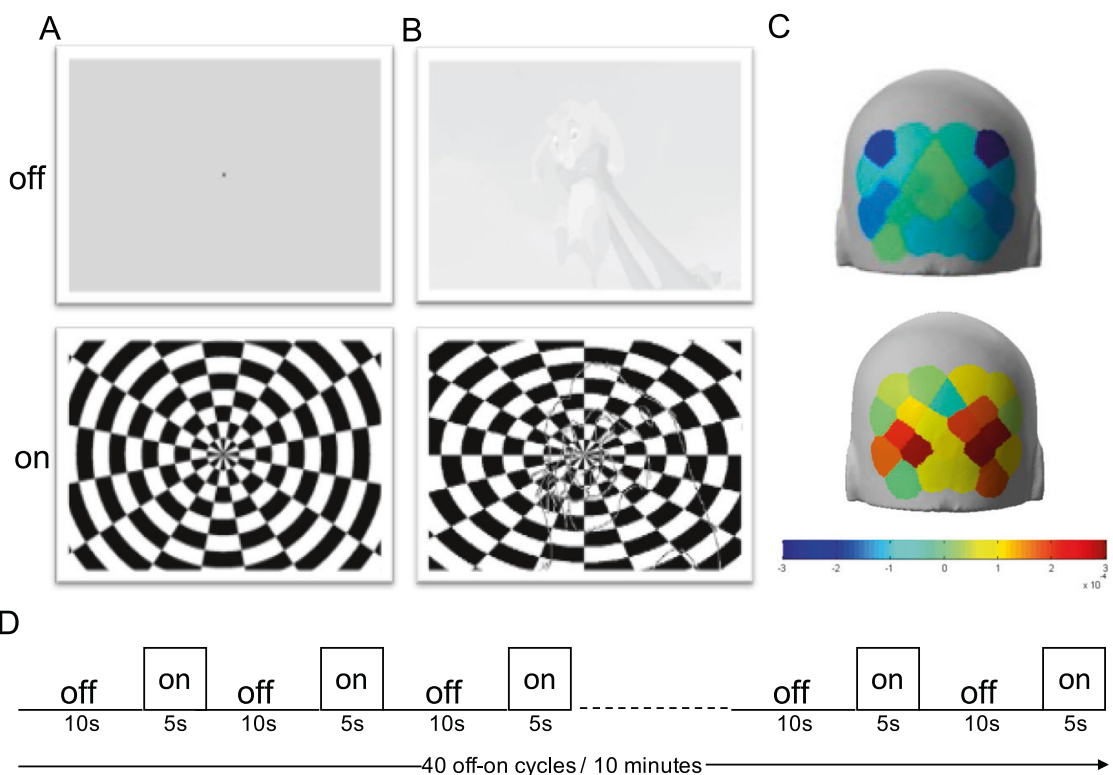

Fig. 1 Visual stimulation and experimental paradigm. A Representative frame of baseline grey screen (upper row, stimulus 'off') and reversing checkerboard (lower row, stimulus 'on') for RS condition. The small black square indicates the fixation point. B Representative frame of low-contrast (20\%) grey-scale baseline animated cartoon (upper row, stimulus 'off') and blended checkerboard-cartoon (lower row, stimulus 'on') for Cartoon-Fixed (CF) and Cartoon Chosen (CC) conditions. C Representative HDR in the occipital cortex during the stimulus 'off' (upper row) and stimulus 'on' activation phase (lower row) according to the output of nirsLAB software. The Look Up table is reported under the images. D Experimental protocol showing that the cycles of visual stimulation were structured in blocks of 40 trials ( 20 trials with the reversing checkerboard and 20 trials with the 'mock' stimulus) for a total duration of $10 \mathrm{~min}$.

Our data showed a significant activation of the visual cortex, with a prominent change of $\mathrm{THb}, \mathrm{OHb}$, and $\mathrm{DHb}$ concentration in response to the $S$ with respect to the blank for all conditions tested (Fig. 3A-C; see Table S1 for statistical details). The amplitude of elicited cortical responses was comparable following L1, H1, and L2 (Fig. 3D; Table S1), proving that the HDR is independent from the cartoon narrative selected for the baseline and the contrast level of baseline presentation in children as well. Small differences were observed for response latency among L1, $\mathrm{H} 1$, and L2 conditions (Fig. S4B; Table S1). A highly significant pattern of correlations among different HDR indexes recorded in the diverse conditions was detected (Fig. S5; see Table S1 for statistical details).

In agreement with previous literature [79], a maturational trend of cortical responsivity was recognized, with children showing significantly higher HDR amplitude with respect to adult subjects (Fig. 3E; Table S1). On the contrary, age-dependent effects were not identified for response latency (Fig. S4C; Table S1).

These findings establish a novel method for measuring visuallyevoked cortical activity with fNIRS that ensures an elevated compliance of young subjects and high-quality reliability of measurements, suggesting a valuable tool for studying visual cortical processing in typically developing children, but also in clinically relevant populations.

\section{Negative correlation of HDR amplitude with AQ score}

Despite no effects detectable in adults (Fig. 4A-C; Table S1), the amplitude of visual responses was highly correlated to $A Q$ scores in children (Fig. 4D, E; see Table S1 for statistical details). Consistent with a recent work [57], the correlation was specific for $\mathrm{THb}$, with higher $\mathrm{AQ}$ score being associated with a lower amplitude of $\mathrm{THb}$ visually-evoked signals (Fig. 4D, E).

Interestingly, HDR amplitude was especially linked to social and communication autistic traits (Fig. 5, S6; Table S1): indeed, assessing separately the five $A Q$ subscales [77] we found a significant correlation of $\mathrm{THb}$ and $\mathrm{OHb}$ with the Social Skills subscale (AQ_S, Fig. 5A, B), while only THb modulation was related to the Communication subscale (AQ_C, Fig. 5C, D) and no significant interaction was observed testing the other three $A Q$ subscales (AQ_A, AQ_D, and AQ_l; Fig. S7). Given the reliability across different visual tasks ( $\mathrm{L} 1$ and $\mathrm{H} 1$ ), the strongest interaction was between $\mathrm{THb}$ and $\mathrm{AQ}$ S (Fig. 5A, B).

\section{DISCUSSION}

We measured hemodynamic responses in the occipital cortex while subjects viewed a reversing checkerboard pattern on a gray isoluminant baseline or the same stimulus blended with a commercial animated cartoon. In all participants, the patterned stimulus elicited a significant change of cortical $\mathrm{Hb}$ (upwards for $\mathrm{THb}$ and $\mathrm{OHb}$, down for $\mathrm{DHb}$ ) independently from the reference baseline, while no response was detected following blank presentation. Since HDR consists of an initial increase in oxygenrich blood followed by a smaller depletion of deoxy-hemoglobin, with a tight interrelation among total, oxygenated, and reduced $\mathrm{Hb}$ levels, reporting all data allows for a more accurate physiological interpretation of the results [78].

Interestingly, the level of occipital cortex activation did not depend on either the movie selected as reference baseline or the baseline contrast, with only a slight reduction of $\mathrm{OHb}$ and $\mathrm{DHb}$ change in response to the stimulus blended to animated cartoons with respect to the classic RS condition. These data demonstrate the reliability of this novel procedure with high entertaining and ecological value in eliciting cortical activity. Thus, our approach might be helpful for studying cortical function in children with an atypical trajectory of brain development, commonly showing a reduced compliance in experimental environments.

The magnitude of HDR modulation, and in particular of $\mathrm{THb}$, was inversely correlated with AQ scores in children. Indeed, we found that the higher were the AQ scores of subjects the lower was the amplitude of $\mathrm{THb}$ response to visual stimulation, suggesting that visually-evoked fNIRS responses are able to capture the dimension of autistic traits in the general young population. Our findings are consistent with previous studies 

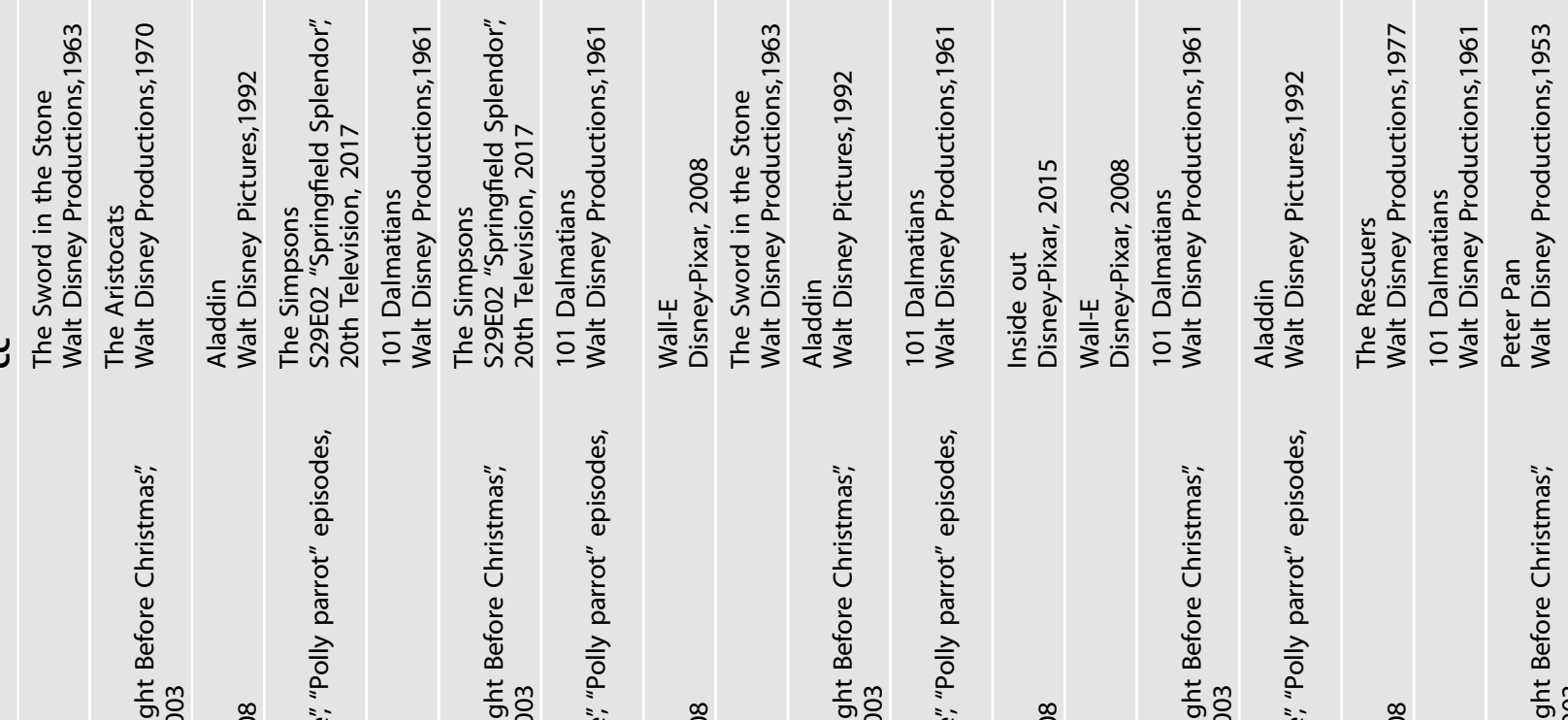

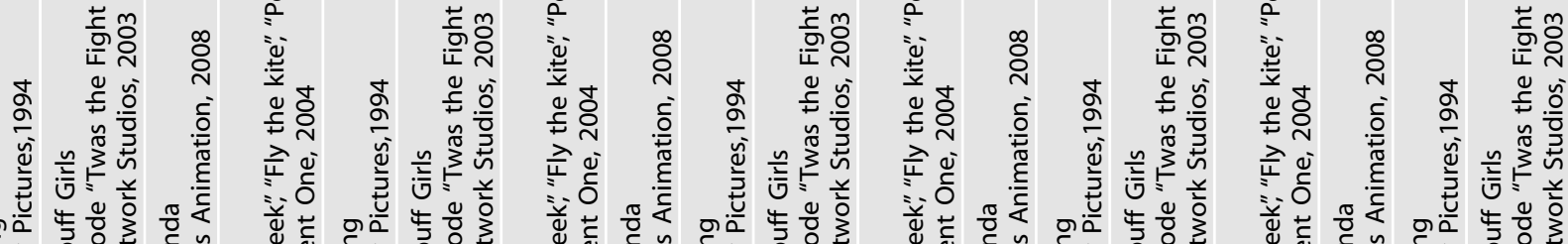

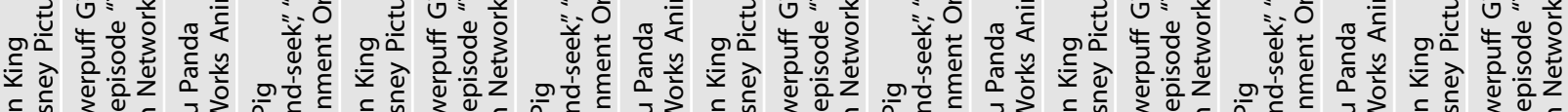

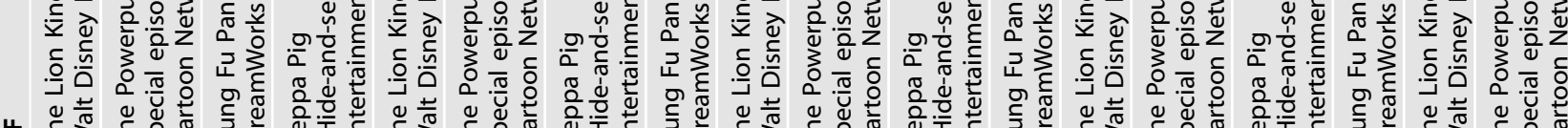

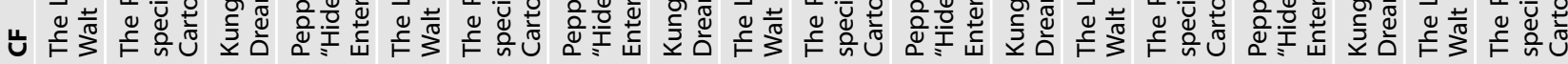

dia

के-

dु.

岁- o. m o

a

密

$g=\therefore=m$

$\frac{\pi}{\mathrm{N}}$

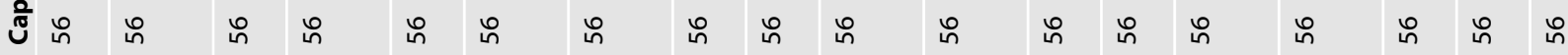

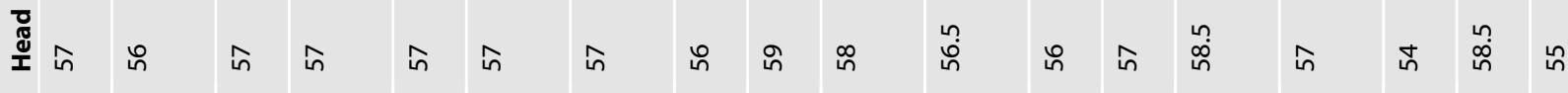

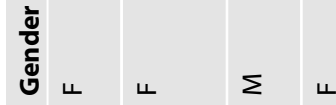

\&্்

毫 


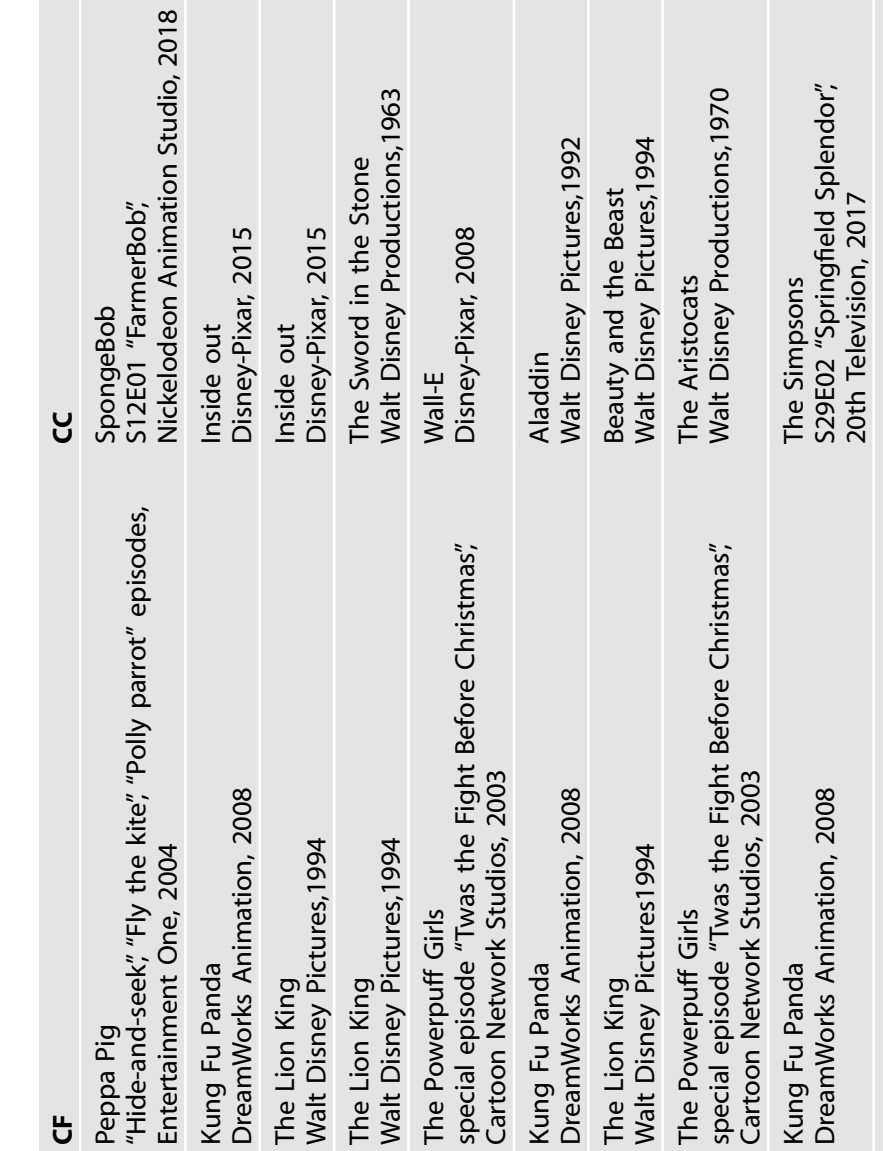

产

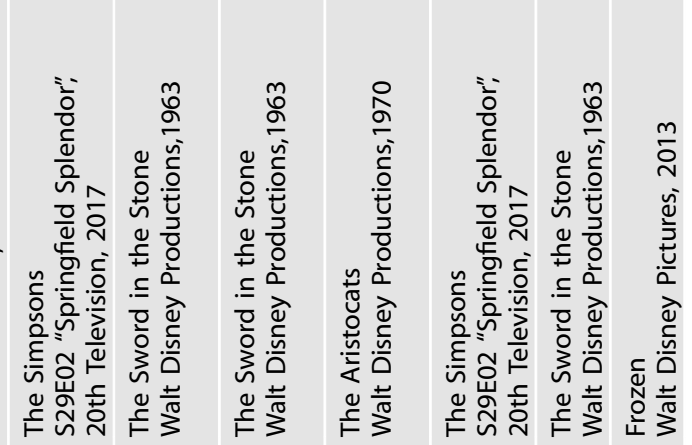

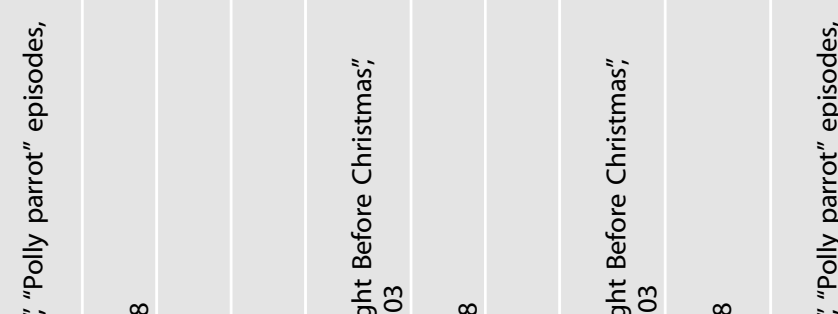

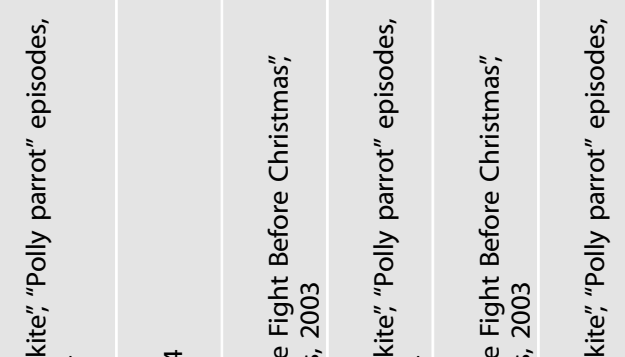

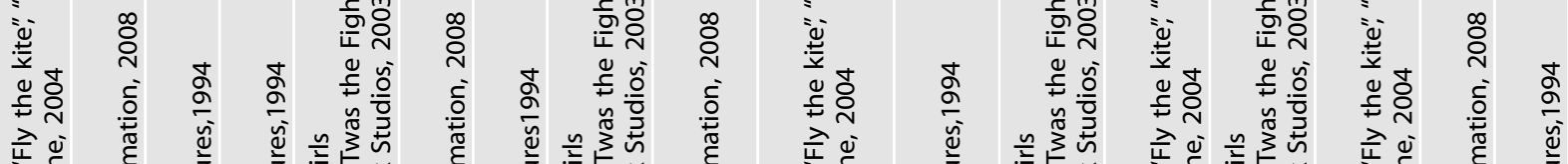

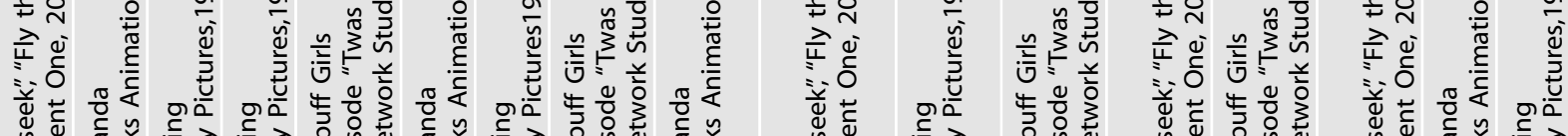

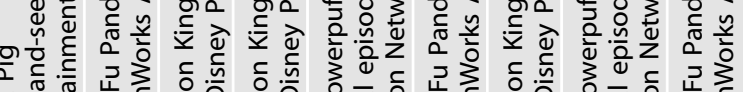

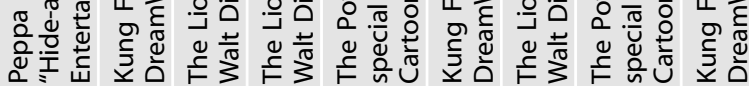

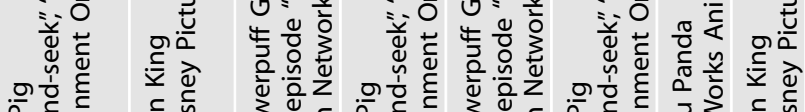

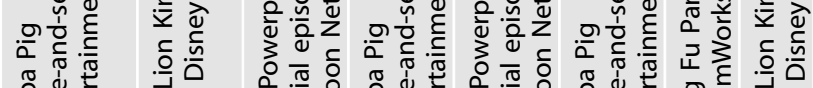

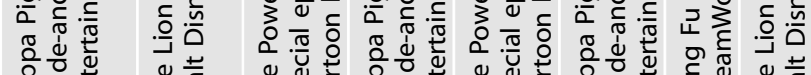
वे

वे

¿

$u^{\prime} u^{\prime}$

a

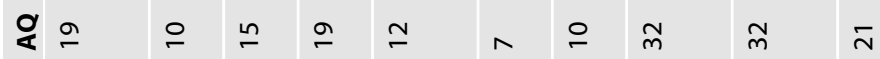

$\frac{\pi}{n}$

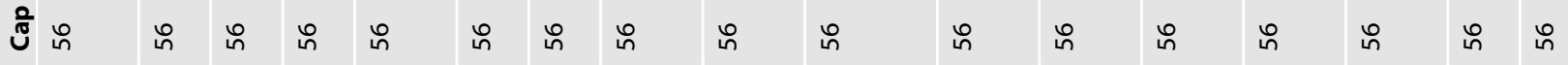

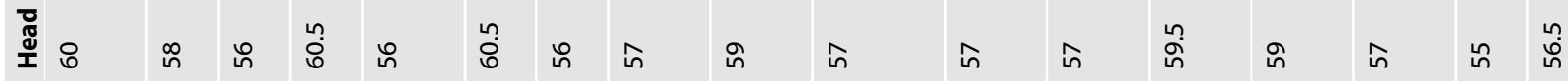

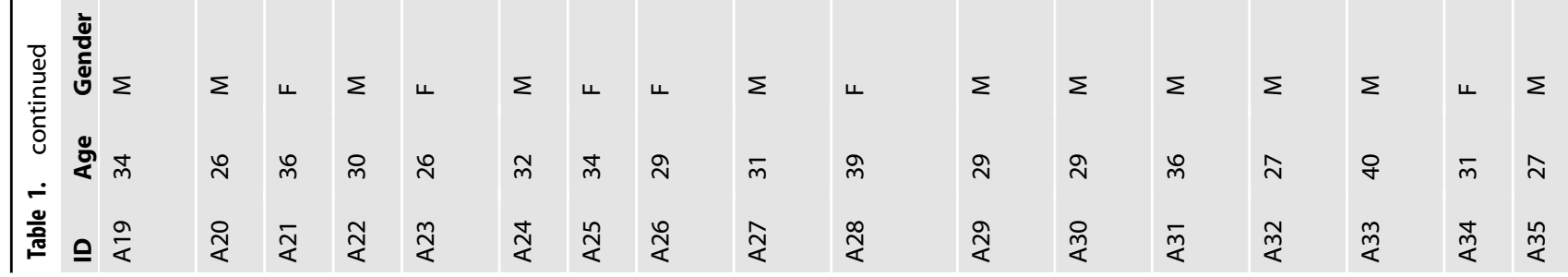


showing that cortical activation measured with fNIRS and the performance in visual psycophysics negatively reflected ASD symptom severity $[45,46,71,72,80-83]$. Moreover, these data reinforce the concept that $\mathrm{THb}$ changes could provide richer discriminative information for classifying between typically developing children and ASD subjects [57]. In our experiment, the THb index describes about $45 \%$ of the variance in AQ scores. This correlation is remarkably high, considering that it is detected across two separate visual stimulating procedures, i.e., the vision of low- and the high-contrast blended RS-animated cartoons. Since levels of $\mathrm{THb}$ reflect the relative changes of both $\mathrm{OHb}$ and $\mathrm{DHb}$ concentrations over the visual cortex [21], we surmise that the better correlation of $\mathrm{THb}$ with $\mathrm{AQ}$ scores might be due to a combinatory effect of $\mathrm{OHb}$ and $\mathrm{DHb}$ variables. The information about $\mathrm{OHb}$ increase and $\mathrm{DHb}$ reduction for each subject, indeed, converges in the modification of THb levels [78].

A tentative explanation of reduced HDR in children with stronger autistic traits might be found in the difference of perceptual styles in the general population $[84,85]$ : the preference for focusing on local details vs. the global stimulus configuration, indeed, is a defining feature of ASD [86] and locally centered perception could be less effective in activating the neural circuits of visual cortex. Interestingly, a recent study established a vascular link to ASD, showing early dysfunction of endothelial cells and impaired endotheliumdependent vasodilation in a mouse model of 16p11.2 deletion [87]. Since the HDR measured with fNIRS strongly relies on neurovascular coupling, this suggests that lower neurophysiological activity may stem in part from endothelial-dependent vascular factors.

In contrast, we failed to detect any correlation between HDR and $\mathrm{AQ}$ scores in adult subjects. This is likely due to the different output range of variables measured in children and adult participants. Accordingly, the 4-point Likert scale used for questionnaires in child population changes the range of $A Q$ scores from $0-50$ to $0-150$, potentially revealing a different variability of inter-individual traits. Moreover, the maturation of neural and vascular networks over brain development affects the pattern of hemodynamic responses [88, 89]. Indeed, our data showed that the amplitude of fNIRS visually-evoked responses is significantly higher in children compared to adults, with a parallel broader distribution of recorded signals.

Although the autistic questionnaire for children is well-validated, showing good test-retest reliability and high internal consistency, not all items have the same validity and factor analysis identified five subscales, named 'Social Skills', 'Communication', 'Attention to Detail', 'Imagination' and 'Attention Switching' [77]. We observed variability in the strength of correlation between HDR and $A Q$ subscales, with the highest correlation for the 'Social Skills' and 'Communication' subscales. Interestingly, 'Social Skills' and 'Communication' are the subscales with higher construct validity performance in differentiating individuals with or without ASD $[90,91]$, while 'Attention to Detail' was the poorest classifying domain [92]. It is also worth stressing that among the items composing the 'Attention to Detail' subscale, only four actually relate to sensory perception (e.g., 'My child usually notices details that others do not'), the others being more focused on cognitive functions (e.g., 'My child is fascinated by numbers'). Accordingly, the first pilot report of the $A Q$ questionnaire showed lower internal consistency (measured by Cronbach's alpha coefficient) of 'Attention to Detail' items compared to other subscales [76].

Although primarily affecting social functioning, there is a growing body of evidence showing that ASD is also associated with abnormalities in multiple sensory domains, fluctuating between hyper- and hypo-sensitivity to sensory stimuli [80, 93]. In addition to a higher incidence of refractive errors and strabismus [94], anomalies in visual processing, visual attention, and visualmotor integration have been described in ASD population $[71,72,74,80,95]$. Interestingly, sensory symptoms are correlated with the severity of the disorder, at least in children [96]. Moreover, 

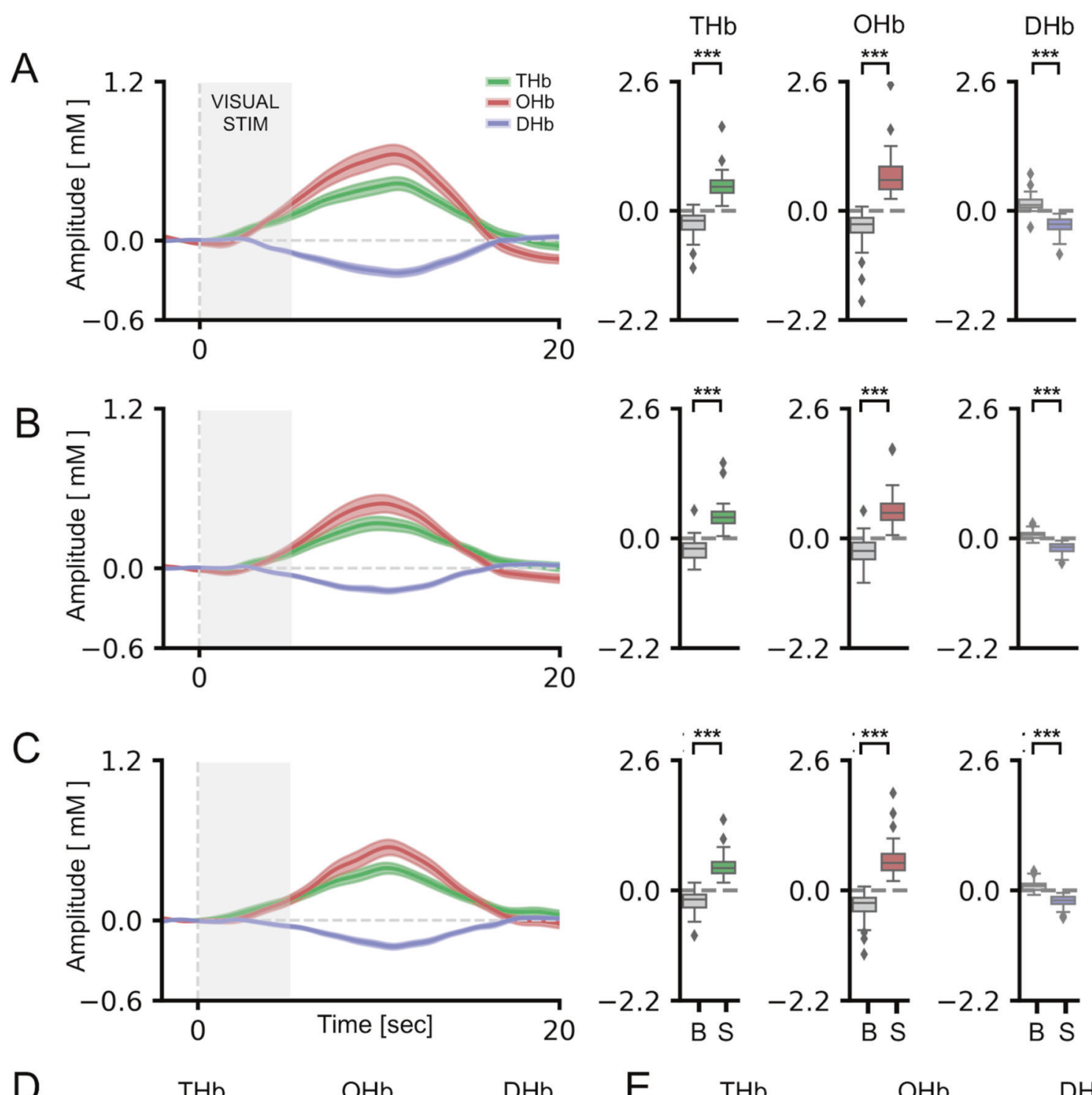

D

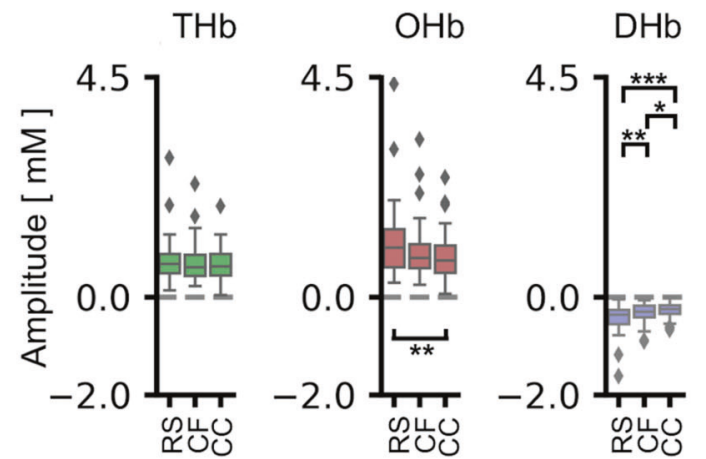

E THb

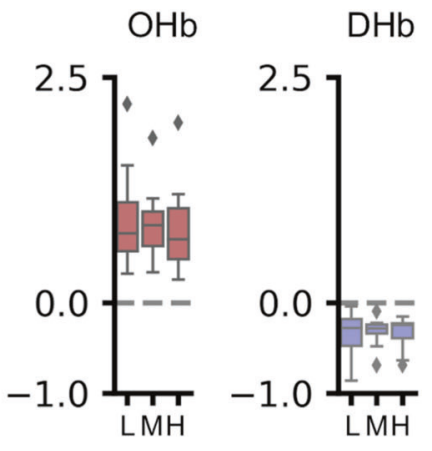

Fig. 2 HDR was reliably detected in adults using both RS and blended RS-animated cartoons. For all panels, values in the $y$-axis are multiplied for $10 \wedge 4$. A On the left, the average time course for THb (green line), $\mathrm{OHb}$ (red line), and DHb (blue line) in response to the Radial Simulus (RS) are shown. The three plots on the right depict the average peak response to the stimulus (S) vs. the blank (B) across all the adult subjects. The stimulus-driven signal was significantly different from the blank for all the conditions (t-test, $p<0.001$ for all comparisons). B Same plots as above for the Cartoon Fixed (CF) condition. On the left, the average time course of the evoked HDR is depicted. On the right, the graphs showed that the HDR amplitude was significantly higher in response to $\mathrm{S}$ with respect to $\mathrm{B}$ for $\mathrm{THb}, \mathrm{OHb}$, and $\mathrm{DHb}(\mathrm{t}-\mathrm{test}, p<0.001$ for all comparisons). C Cartoon Chosen (CC) condition. Also in this case the S elicited significantly higher responses for $\mathrm{THb}, \mathrm{OHb}$, and $\mathrm{DHb}$ with respect to the $\mathrm{B}$ (t-test, $p<0.001$ for all comparisons). D Comparison among different visual stimulations shows no differences in evoked amplitudes for $\mathrm{THb}$, whereas a significant difference was detected between RS and CC for OHb (One-way RM ANOVA, $p<0.01$, post hoc BHFDR, RS vs. CC $p<0.01$ ) and a more complex pattern of differences emerged for DHb (One-way RM ANOVA, $p<0.001$, post hoc BH-FDR, RS vs. CF $p<0.01$, RS vs. CC $p<0.001$, CF vs CC $p<0.05$ ). E No differences of evoked responses were detected with different contrast levels of the baseline movie (L: low, M: medium, $\mathrm{H}$ : high). For statistical metrics and details, refer to Table S1. Data are shown as average \pm s.e.m. ${ }^{*} p<0.05$; ${ }^{* *} p<0.01$; ${ }^{* * *} p<0.001$. 


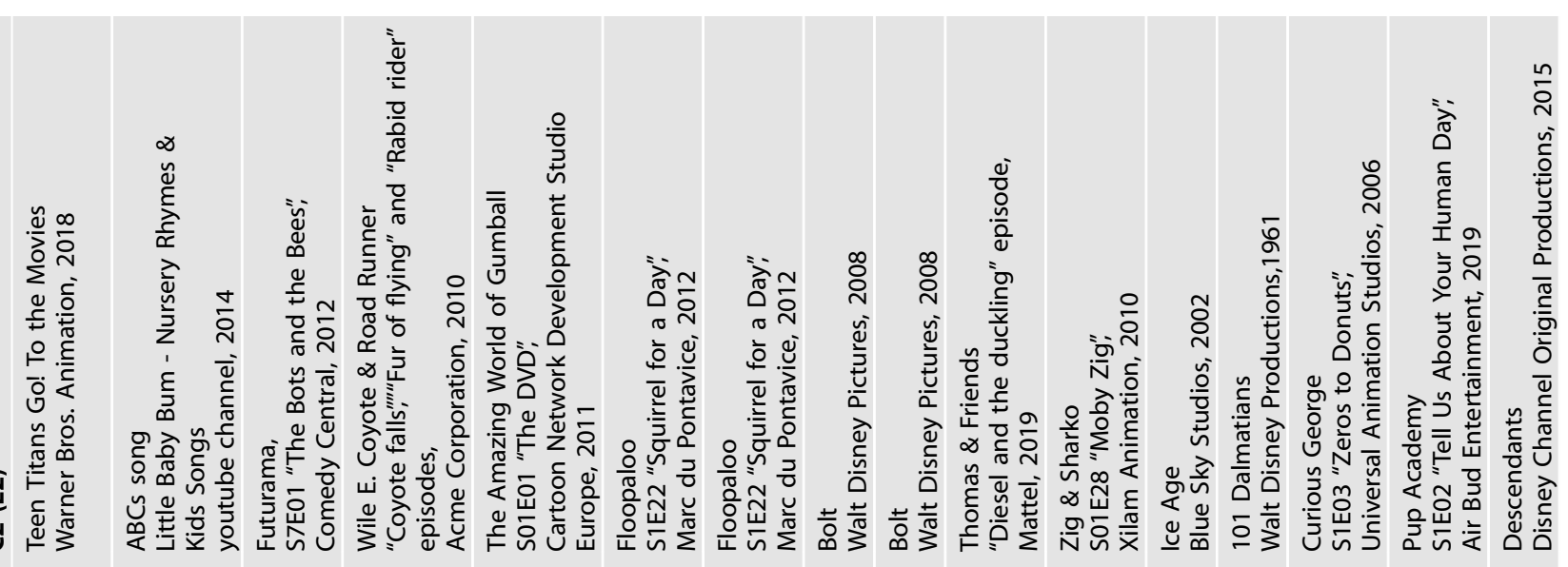

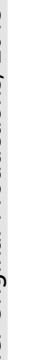

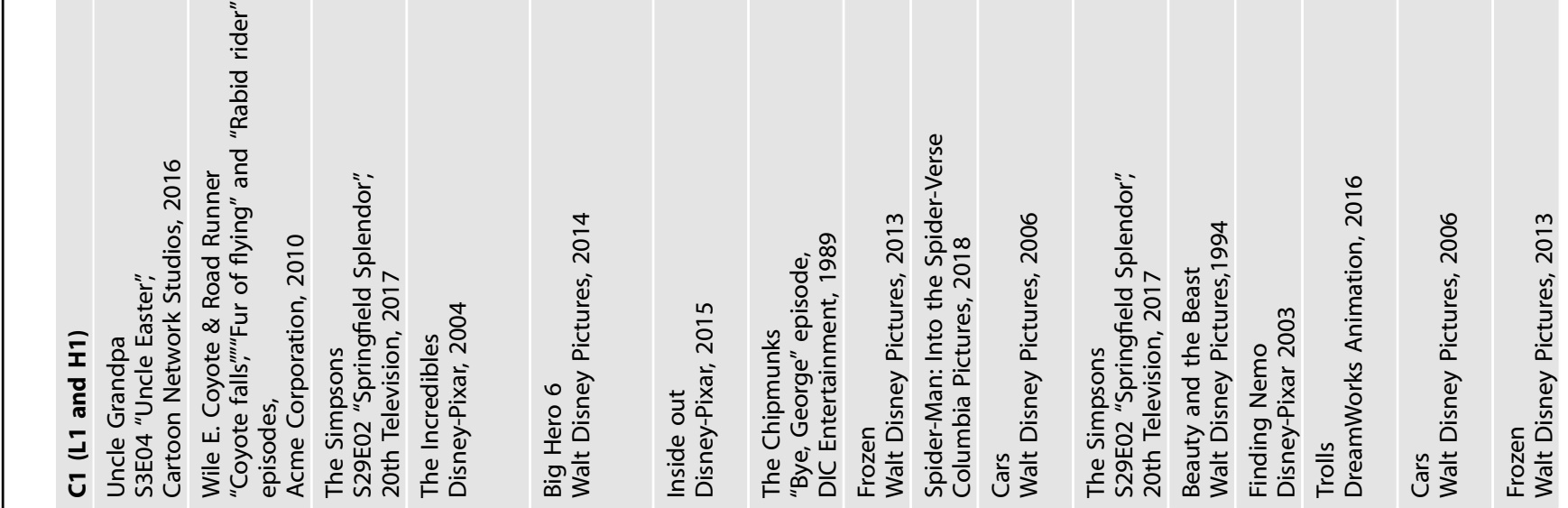
永。

वे

ब

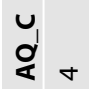
安-

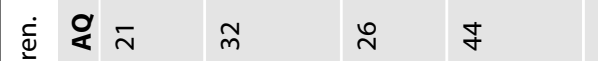
产 $\frac{\mathrm{N}}{\mathrm{N}}$

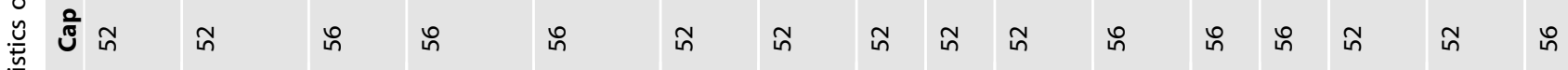

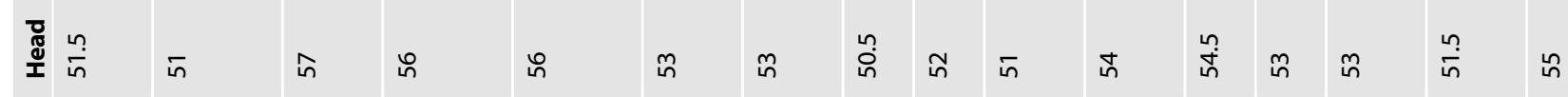
$\frac{0}{\frac{1}{0}} \frac{0}{\frac{\pi}{0}}$<smiles>C1=C[C+]2CCC(C1)C2</smiles> 


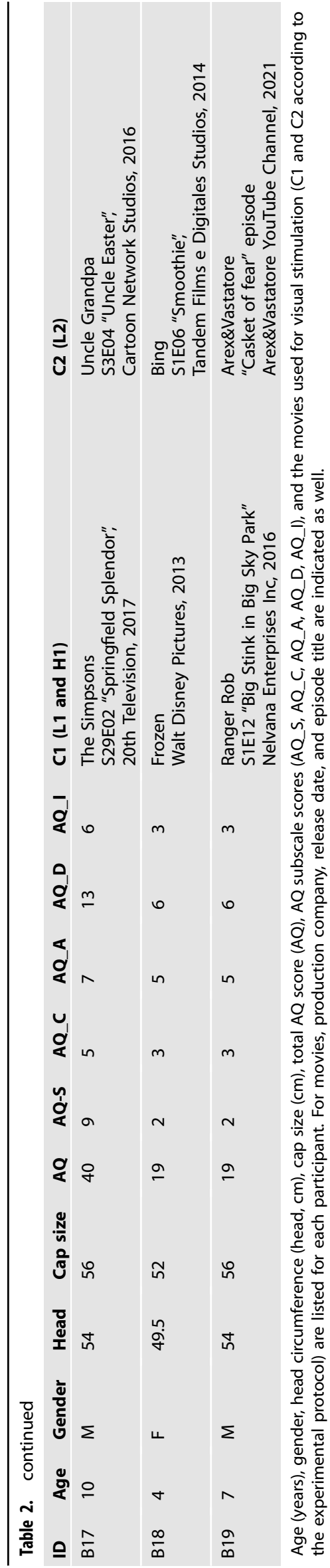

commonly observed alterations in social skills might have a visual component [80] and perception deficits could impact with cascading effects on the maturation of cognitive and social domains [95].

It has been recently suggested that an early assessment of pupil size modulation and visual behavior might improve the diagnostic process of ASD [74, 84, 95, 97]. Currently, ASD diagnosis and follow-up almost entirely rely on phenotypic information collected via clinical measures and parental input that are highly prone to subjective bias [98]. Moreover, the late appearance of some behavioral autistic traits often delays the diagnosis until midchildhood [99, 100]. Thus, the identification of solid brain biomarkers early predicting ASD pathophysiology is a critical step to anticipate tailored interventions, leading to better outcomes for patients and possibly even the prevention of certain behaviors typically associated with ASD. Objective biomarkers have also the potential to be helpful in the management of patients, allowing the classification of disease severity and monitoring response to treatments [14]. A recent systematic review highlighted that both functional and structural neuroimaging features might predict ASD diagnosis in the early pre-symptomatic period [101, 102], but further studies are needed to validate the promising performance of such biomarkers [14]. Lately, resting-state fNIRS measurements have been suggested as candidate biomarkers for ASD $[32,57,59,60]$. As stressed above, fNIRS offers significant advantages with respect to other neuroimaging tools, including non-invasiveness, ease of use, no need of sedation, tolerance to movements, and portability, making it a child-friendly approach. However, the extraction of metrics with diagnostic value from resting-state recordings involves complex algorithms.

In contrast, our analysis of visually evoked responses is quick, easy, and requires only that children pay attention to a short movie of their choice. Since our stimulating strategy has been studied to optimize the compliance of young subjects, we believe that our results might set the background for testing fNIRS visual measurements in ASD individuals. Moreover, screening for autistic traits in the general population may be helpful in epidemiological research because it may provide a large sample size to investigate the correlation between autism phenotype severity and other pathophysiological processes [91].

Although our study provides a first step towards the use of fNIRS for empowering early detection of autistic traits, some limitations need to be discussed. First, the quantification of $A Q$ scores for children according to parent questionnaires might introduce a response bias in the dataset [103]. Despite the high test-retest and reliability coefficients of AQ-Child [77], future studies might examine autistic traits in the child general population with an integrated approach consisting of direct behavioral observation and administration of multidimensional questionnaires. Moreover, testing a larger and gender-balanced sample will allow not only to confirm the validity of our results, but also to potentially highlight gender differences in fNIRS measurements and to stratify the child population in different age groups. Finally, combined fNIRS-EEG recordings are needed to dissect whether the sensitivity of fNIRS to autistic traits is determined by neural or vascular processes.

\section{MATERIALS AND METHODS \\ Subjects}

We recruited a total of 40 adult subjects (20 women, age: $31.05 \pm 3.94$ (SD) years) and 19 children ( 5 girls, age: $7.20 \pm 3.01$ (SD) years). All participants reported normal or corrected-to-normal vision and had no diagnosed neuropsychiatric condition. Experimental procedures on children were authorized by the Regional Pediatrics Ethics Board (Comitato Etico Pediatrico Regionale-Azienda Ospedaliero-Universitaria Meyer-Firenze, Italy; authorization number 201/2019) and were performed according to the declaration of Helsinki. Written informed consent was obtained from all 

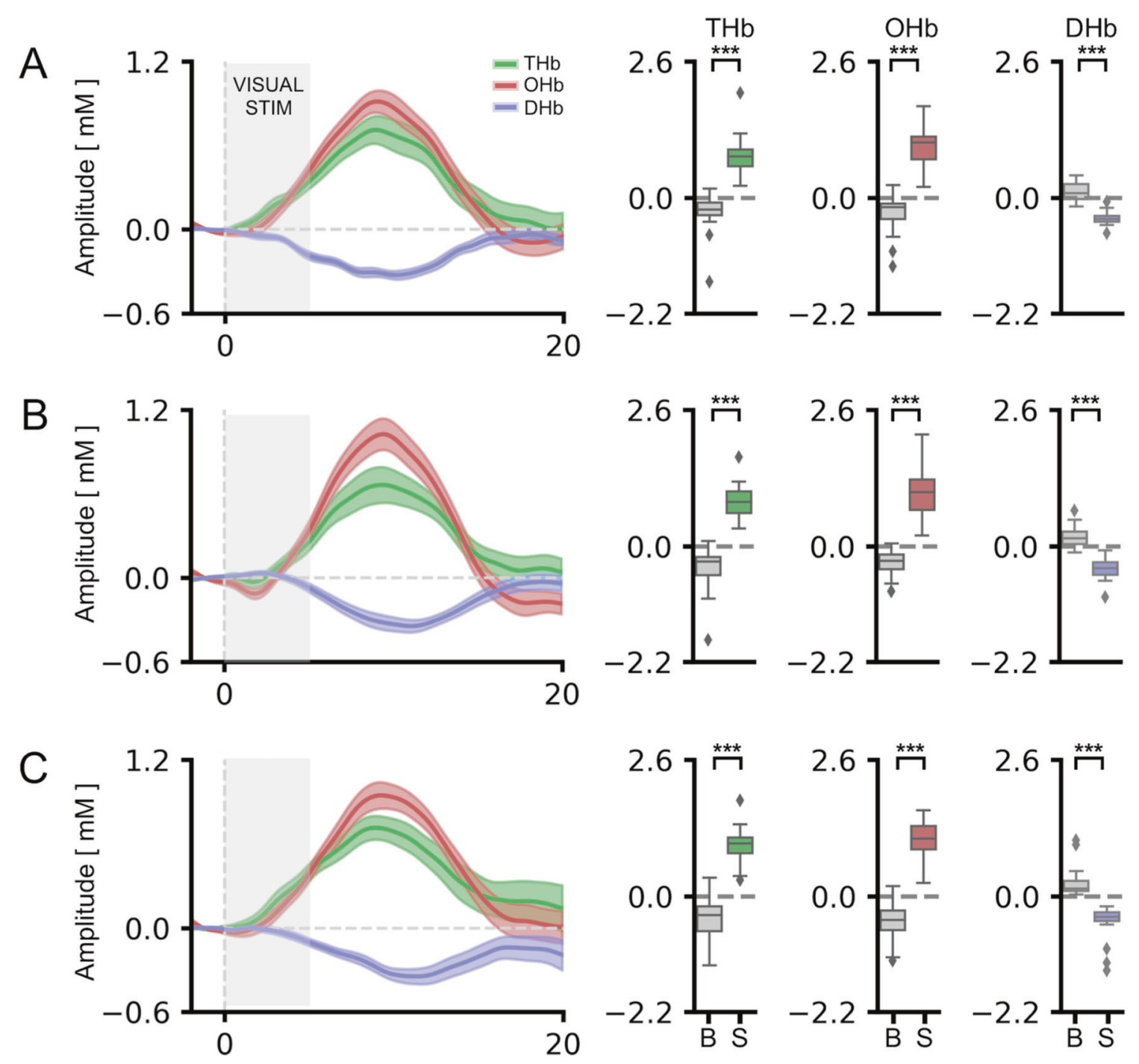

$\mathrm{D}$

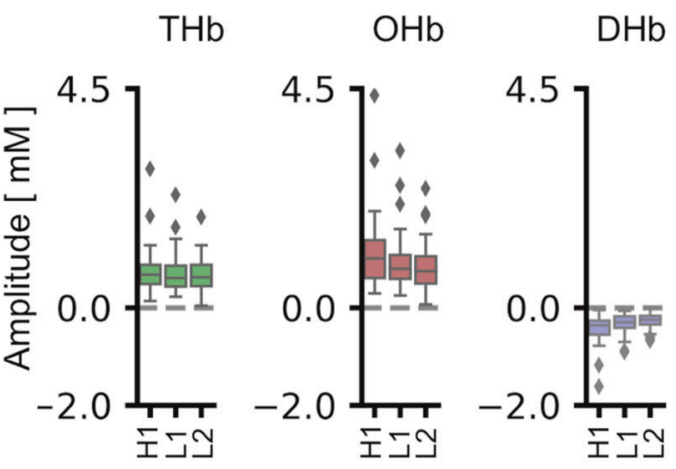

adult participants and from the parents of each child, authorizing the use of anonymized data for research purposes. Assent was also obtained from the children involved in the study before participation.

\section{AQ score}

Adult participants filled in the Autistic-Spectrum Quotient (AQ) questionnaire, a 50-items self-administered report validated for the Italian version [76, 104]. The items consist of descriptive statements assessing personal preferences and typical behavior. For each item, participants respond on a 4-point Likert scale: "strongly agree", "slightly agree", "slightly

E THb

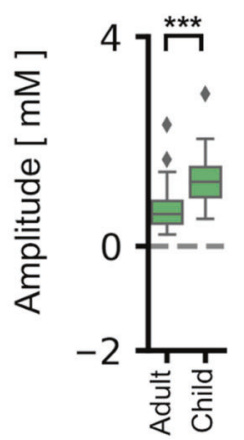

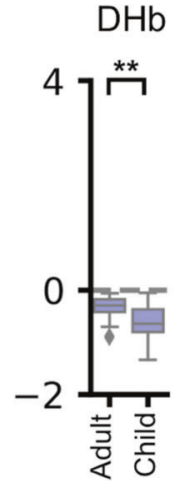

disagree", and "strongly disagree". The items are grouped in five subscales: Social Skills, Communication, Attention to Details, Imagination, and Attention Switching. All the questionnaires were scored by a neuropsychiatrist blinded to subject data: 1 point was assigned when the participant's response was characteristic of ASD (slightly or strongly), 0 points were attributed otherwise. Total scores range between 0 and 50 (0-10 for each subscale), with 32 being the clinical threshold for autism risk [76]. No subjects scoring above 32 points were recorded. The mean (minmax) of the scores was 15.1 (3-32) with SD of 6.5 (Table S2). The children's version of Autism-Spectrum Quotient (Italian version of AQ-child) was completed by parents [77]. This version of the $A Q$ questionnaire includes 
Fig. 3 HDR signal was reliably detected in children using blended RS-animated cartoons with low and high contrast. For all panels, values in the $y$-axis are multiplied for $10^{\wedge} 4$. A On the left, the average time course for $\mathrm{THb}$ (green line), $\mathrm{OHb}$ (red line), and $\mathrm{DHb}$ (blue line) in response to high-contrast $(80 \%)$ blended RS-animated cartoons is shown $(\mathrm{CH} 1)$. On the right, the graphs represent the average amplitude of the evoked HDR following the stimulus $(\mathrm{S})$ and the blank (B). A significantly different response to $\mathrm{S}$ with respect to the $\mathrm{B}$ was detectable for all metrics ( $\mathrm{t}$ test, $p<0.001$ for all comparisons). B The average time course of the HDR to low-contrast (20\%) blended RS-animated cartoons is shown (CL1). Here, the baseline cartoon is the same as the experiment described in panel (A), but a different part of the movie was used. THb, OHb, and $\mathrm{DHb}$ showed a significantly higher deflection to the $\mathrm{S}$ with respect to the $\mathrm{B}$ in this condition as well (t-test, $p<0.001$ for all comparisons). $\mathrm{C}$ On the left, the average time course of HDR following the second low-contrast blended RS-animated cartoon selected by the subject (CL2). On the right, the analysis of peak amplitudes revealed significantly higher responses during $\mathrm{S}$ compared to $\mathrm{B}$ for $\mathrm{THb}, \mathrm{OHb}$, and $\mathrm{DHb}$ (t-test, $p<$ 0.001 for all comparisons). D Comparison among different contrast levels of the baseline cartoon revealed no differences in the amplitude of HDR. E Response amplitudes for low-contrast blended RS-animated cartoons in adults and children. More specifically, we compared the response to CF condition of adults with CL1 condition for children. The average amplitude of HDR was significantly higher in children (t-test, $p<0.001$ for THb, $p<0.05$ for $\mathrm{OHb}, p<0.01$ for $\mathrm{DHb}$ ). For statistical metrics and details, refer to Table S1. Data are shown as average \pm s.e.m. ${ }^{*} p<0.05 ;{ }^{* *} p<0.01 ; * * * 0.001$.

50 items as well, grouped in the same subscales described above, and parents were required to report for each statement the degree of consistency with their child's behavior. Scores range from 0 to 150 (0-30 for each subscale), since the response scale is treated as a 4-point Likert scale with 0 representing definitely agree; 1 slightly agree; 2 slightly disagree; and 3 definitely disagree. Items were reverse-scored as needed. The threshold score is 76 [77]. All subjects scored below 76 points. The mean (min-max) of the scores was 32.1 (17-49) with SD of 10.7 (Table S3).

\section{Apparatus and montages}

To measure changes in total $\mathrm{Hb}(\mathrm{THb})$ concentration and relative oxygenation levels $(\mathrm{OHb}$ and $\mathrm{DHb})$ in the occipital cortex during the task, we used a continuous-wave NIRS system (NIRSport $8 \times 8$, NIRx Medical Technologies LLC, Berlin, Germany). Our NIRSport system consists of eight red light-sources operating at 760 and $850 \mathrm{~nm}$, and seven detectors that can be placed into a textile EEG cap (EASYCAP, Herrsching, Germany), forming an array of 22 multidistant channels [105]. Textile EEG caps of different sizes were used. The probe arrangement was fixed in each of the caps using grommets, optode stabilizers, colored labels, and holders in order to assure comparable probe mapping over all subjects. For data recording, the Aurora Software 1.4.1.1 (NIRx Medical Technologies LLC) was employed. The sampling rate was $10.2 \mathrm{~Hz}$. Visual areas were identified according to the craniocerebral topography within the international 10-20 system and the placement of the optodes was done using fOLD v2.2 [106] and NIRSite 2.0 (NIRx Medical Technologies LLC) softwares. Sources and detectors were symmetrically distributed to define 22 channels around the region of interest, each adjacent pair of sources and detectors defining one channel (min-max source-detector separation: 20-44 mm for adults, 22-30 mm for children; Fig. S2).

\section{Experimental design and visual stimulation}

Prior to the experiment, adult participants (or parents for children) filled in the $A Q$ questionnaire. Then, subjects were asked to sit on a comfortable chair and the fNIRS cap was positioned. Optodes were placed into the cap and the calibration of light coupling between sensors and detectors was performed. All experimental sessions lasted 30-40 min. Visual stimuli were generated using Python 3 and Psychopy3 [107] and displayed with gamma correction on a monitor (Sharp LC-32LE352EWH, $60 \mathrm{~Hz}$ refresh rate, $45 \mathrm{~cd} /$ $\mathrm{m}^{2}$ mean luminance, resolution of $800 \times 600$ pixels) placed $70 \mathrm{~cm}$ from the subject. Cortical hemodynamics in response to full-field, reversing, square wave, radial checkerboard, with abrupt phase inversion (spatial frequency: 0.33 cycles per degree, temporal frequency: $4 \mathrm{~Hz}$; Fig. 1A) was evaluated in the time domain by measuring the peak-to-baseline amplitude and latency. To have an internal control with blank stimulation, we used an event-related design consisting of: (i) 20 cycles of $5 \mathrm{~s}$ stimulus 'on' (reversing checkerboard, $90 \%$ of contrast) followed by $10 \mathrm{~s}$ stimulus 'off' and (ii) 20 cycles of $5 \mathrm{~s}$ mock stimulus 'on' (reversing checkerboard, $0 \%$ of contrast) followed by $10 \mathrm{~s}$ stimulus 'off'. The two stimulating conditions were pseudo randomly interleaved for each subject during the recording. Blocks lasted $10 \mathrm{~min}$ and participants were permitted to take rest between recordings. Figure 1D shows a schematic representation of the experimental procedure. Visual events were synchronized with NIRSport over wireless LAN communication through the Python version of LabStreamingLayer (https://github.com/sccn/labstreaminglayer).

Recordings in adult participants. Experiment 1 for adults (exp1) aimed to understand whether a reliable hemodynamic signal could be recorded in response to the radial checkerboard merged with an animated cartoon. Thus, exp1 started with a 10 min recording using the reversing checkerboard as stimulus 'on' and the gray screen as stimulus 'off' (RS condition), and continued with the vision of two different blended animated cartoons, where the stimulus 'on' was a merge between the reversing checkerboard and the movie, whereas the stimulus 'off' was the gray-scale isoluminant cartoon (CF and CC conditions; Fig. S1). The main purpose of using a cartoon was to increase the entertaining value of visual stimulation. The checkerboard presentation was needed to ensure a standardized episodic stimulation allowing event-related transient analysis. We decided to merge the checkerboard with the movie in order to avoid possibly distracting interruptions of the storyline and to facilitate screen fixation in children. The merging procedure was achieved using Python3 OpenCV [108]. During the appearance of the stimulus each frame was filtered using an automatic Canny edge detection algorithm (https://www.pyimagesearch.com/2015/ 04/06/zero-parameter-automatic-canny-edge-detection-with-python-andopencv/), then the filtered cartoon was blended with the radial checkerboard. Each pixel of the animated cartoon with the same color of the corresponding pixel of the radial checkerboard was inverted, to obtain a fully visible image. The result was a RS with an overlayed cartoon frame (Fig. 1B). The first cartoon was randomly selected by the operator within a group of 4 ("The Lion King", "The Powerpuff Girls", "Peppa Pig" or "Kung Fu Panda"; CF), whereas the latter was a free choice of the subject (CC). Exp2, aiming to dissect the contribution of baseline contrast to visual responses, was performed in a subset of adult participants $(n=15)$. Exp2 consisted of 3 consecutive recordings of a CF ("Peppa Pig"; "Hide-and-seek", "Fly the kite", "Polly parrot" episodes) with the modulation of the baseline contrast $(20,40$, and $80 \%$; Fig. S1). The presentation order of different contrast levels was randomly shuffled.

Recordings in children. To confirm that the baseline movie and its contrast do not affect the emergence of visual responses to the radial checkerboard in children, we measured hemodynamic signals in response to 2 different blended RS-animated cartoons freely decided by the subject: cartoon 1 was presented at both low $(20 \%, \mathrm{~L} 1)$ and high $(80 \%, \mathrm{H} 1)$ contrast, while only low contrast was recorded for cartoon 2 (L2; Fig. S1). In this case, the presentation order was decided by the child, in order to maximize subject compliance.

During the experimental sessions, data were quickly analyzed and visualized using nirsLAB software (NIRx Medical Technologies LLC, v2019.4).

\section{Signal processing and statistical analysis}

Data preprocessing was completed using the Homer3 package (v1.29.8) in MATLAB (R2020a). We created a processing stream tailored on recent guidelines for the analysis of fNIRS data [25]. First, the raw intensity data were converted to optical density (OD) changes ( $h m R_{-}$Intensity2OD). Then, channels showing very high or low optical intensity were excluded from further analyses using the function $h m R \_$PruneChannels (dRange: 5e-04-1e +00 , SNRthresh: 2; SDrange: 0.0-45.0). Motion artifacts were then removed by a multistep rejection protocol. After a step of motion artifact detection using the hmR_MotionArtifactByChannel function (tMotion: 1.0, tMask: 1.0; STDEVthresh 13.0; AMPthresh: 0.40), motion correction was performed with a combination of Spline interpolation (hmR_MotionCorrectSpline, p: 0.99) and Wavelet filtering ( $h m R \_$MotionCorrectWavelet, iqr: $0.80)$ functions [25]. The remaining uncorrected motion artifacts were identified using the $h m R$ _MotionArtifactByChannel. A band-pass filter (hmR_BandpassFilt: Bandpass_Filter_OpticalDensity, hpf: 0.01, Ipf: 0.50) was applied to decrease slow drifts and high-frequency noise, and the OD data 


\section{ADULT}

$\mathrm{THb}$

$\mathrm{OHb}$

$\mathrm{DHb}$
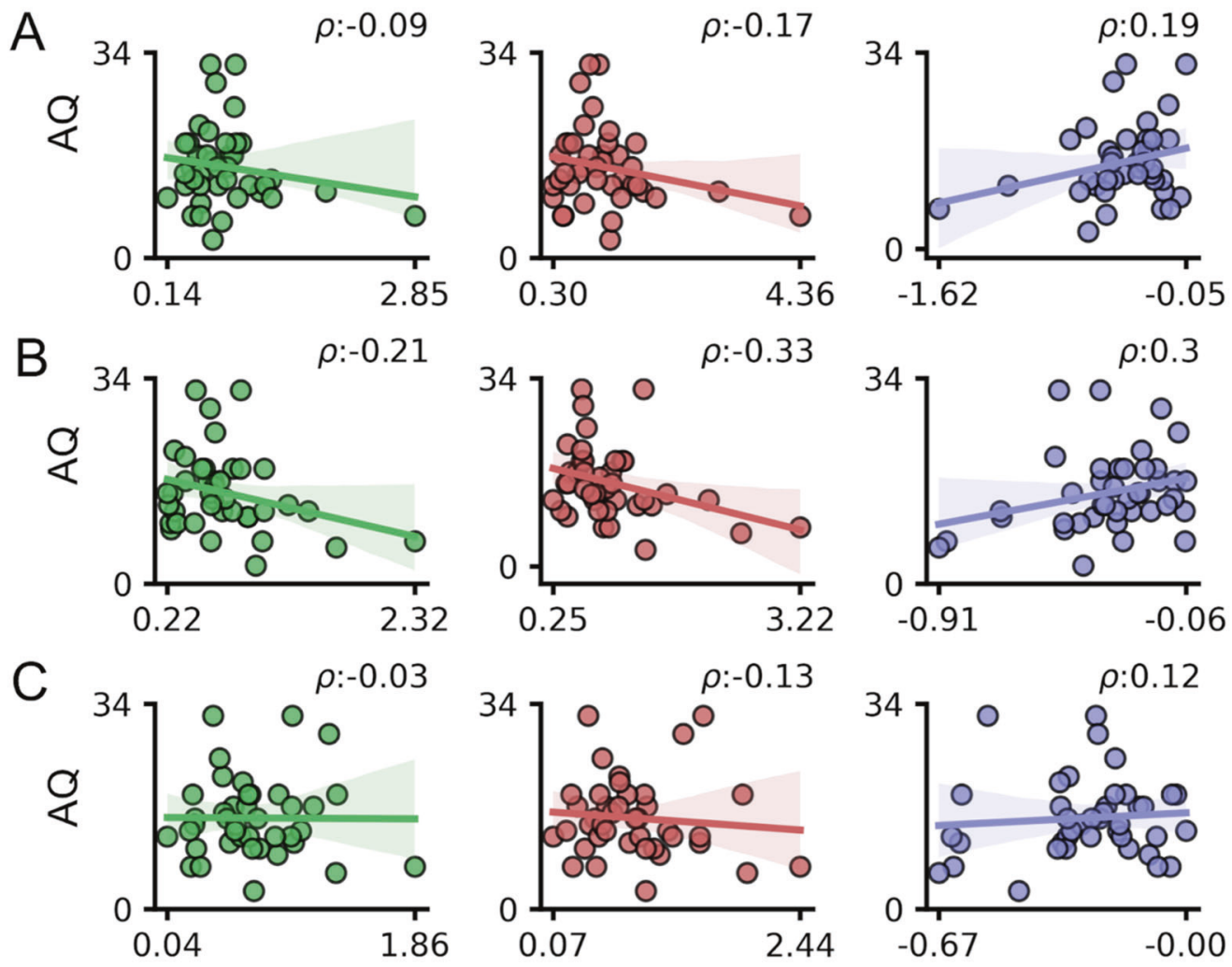

\section{CHILD}
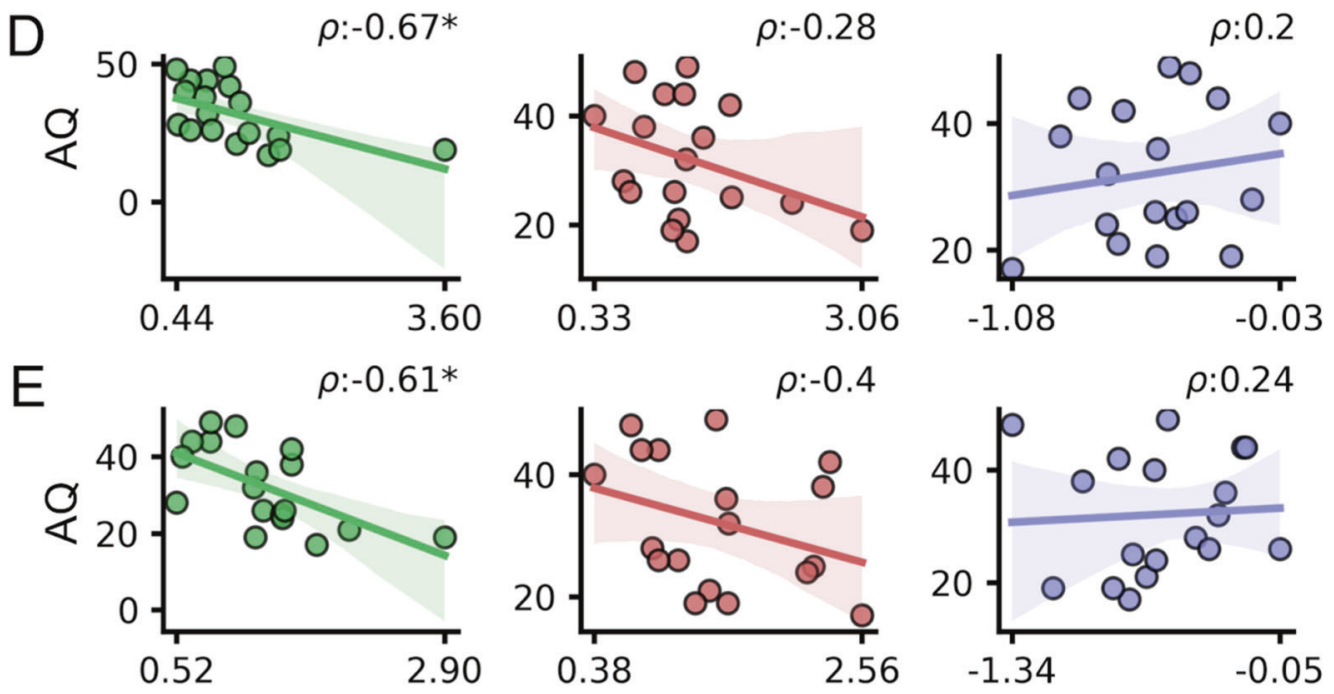

Amplitude [ $\mathrm{mM}$ ]

Amplitude [ $\mathrm{mM}$ ]

Amplitude [ $\mathrm{mM}$ ]

Fig. 4 Correlation between HDR and AQ scores. For all panels, values in the $x$-axis are multiplied for $10 \wedge 4$. The $\rho$ (rho) index in each plot indicates the Spearman correlation value. Correlation between HDR and AQ scores in adults, for amplitudes obtained using RS (A), CF (B), and CC (C). No significant correlations were detected for adult participants. Correlation between HDR and AQ scores in children, for amplitudes obtained using high (D), and low (E) contrast baseline cartoons. A significant correlation was found between THb and AQ scores for both highand low-contrast blended stimuli $(p<0.05$ for both cases). Circles are individual values; lines represent the linear regression model fit and shaded regions are the $95 \% \mathrm{Cl}$. 


\section{$A Q$ Social Skills}

$\mathrm{THb}$

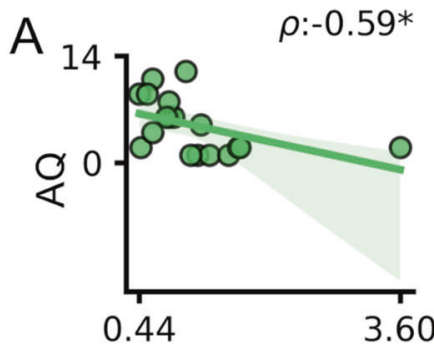

$\mathrm{OHb}$

$\mathrm{DHb}$
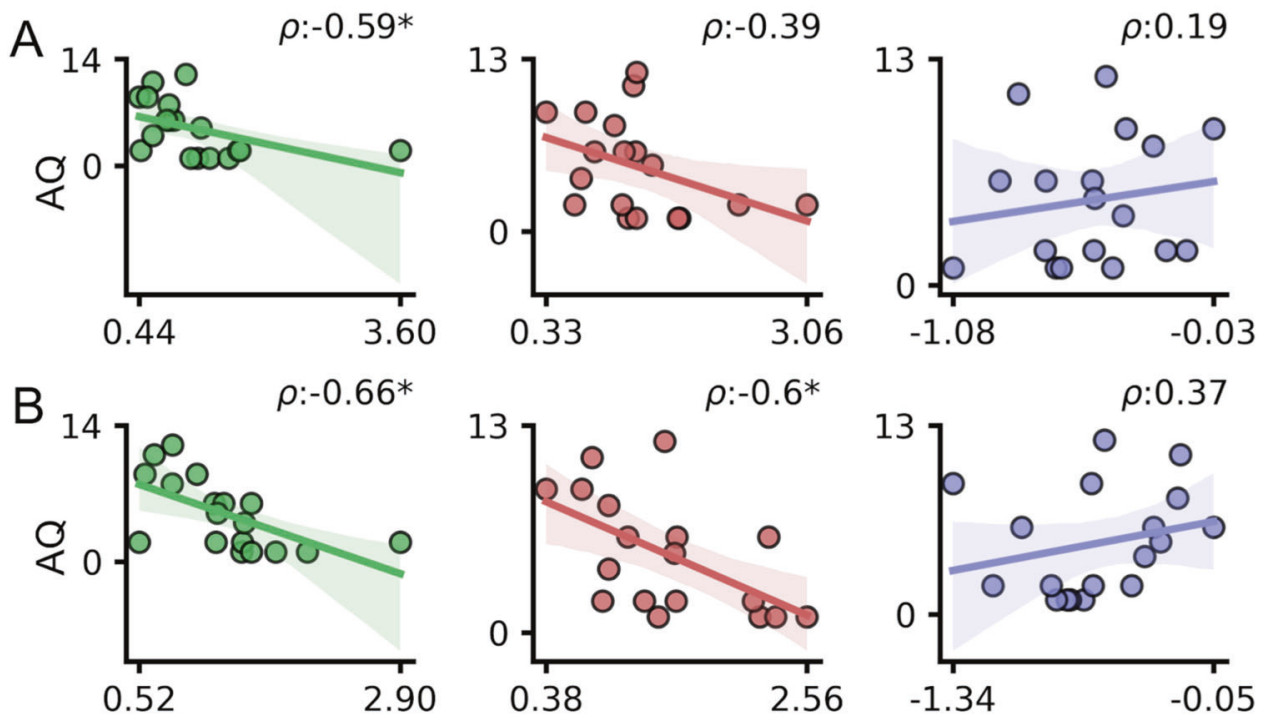

\section{AQ Communication}
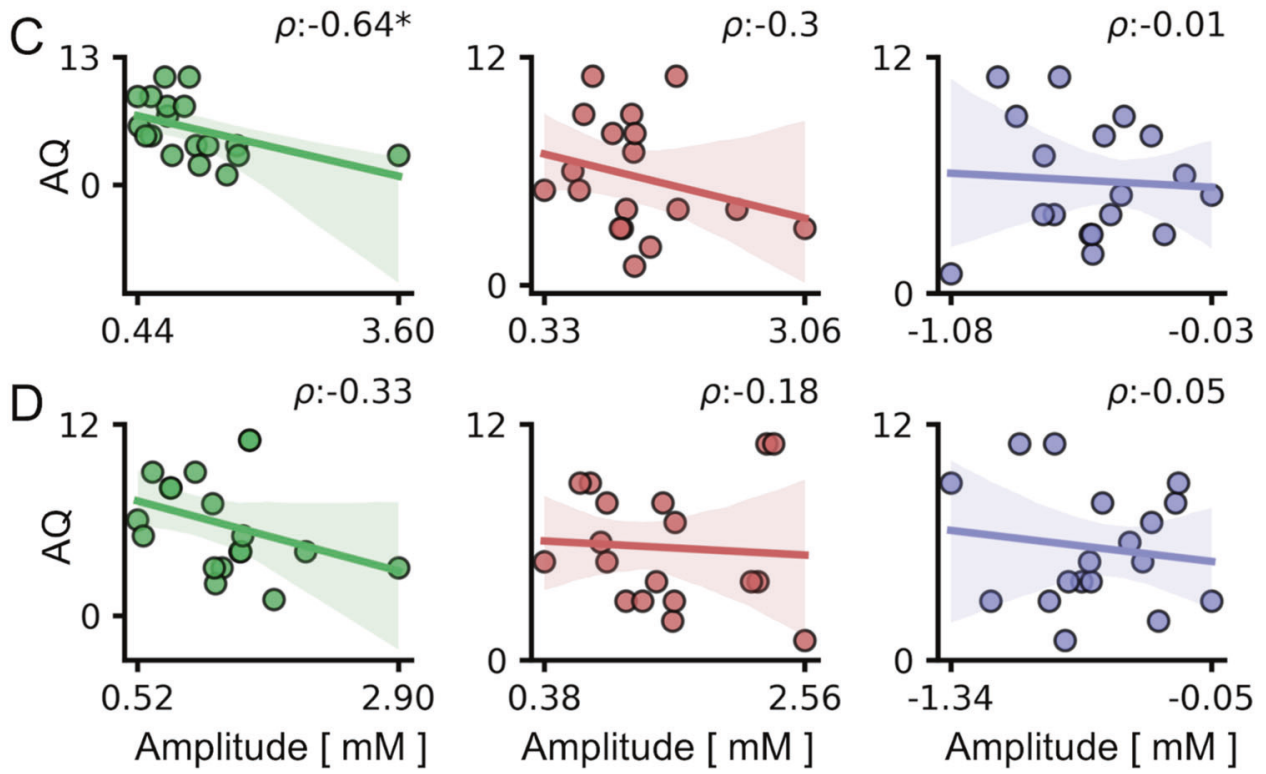

Fig. 5 Correlation between HDR and AQ subscales in children. For all panels, values in the $x$-axis are multiplied for $\times 10 \wedge 4$. The $\rho$ (rho) index in each plot indicates the Spearman correlation value. A, B Correlations between HDR and AQ Social Skills (AQ_S) subscale. A significant correlation between $\mathrm{THb}$ and $\mathrm{AQ} \_\mathrm{S}$ was detected using both high-(A) and low-contrast blended stimuli (B; $p<0.05$ for both cases). In addition, $\mathrm{OHb}$ recorded in response to the low-contrast blended RS-cartoon was significantly correlated with $\mathrm{AQ} S \mathrm{~S}(\mathbf{B} ; p<0.05)$. C, $\mathbf{D}$ Correlations between HDR and AQ Communication (AQ_C) subscale. THb amplitude in response to the high-contrast blended RS-cartoon was significantly correlated with AQ_C $(p<0.05)$. Circles are individual values; lines represent the linear regression model fit and shaded regions are the $95 \% \mathrm{Cl}$.

were converted to $\mathrm{Hb}$ concentration changes using the modified Beer-Lambert law ( $h m R \_O D 2 C o n c$, ppf: 1.01 .01 .0 ). Finally, trials of each subject were block-averaged for every stimulating condition and channel (hmR_BlockAvg: Block Average on Concentration Data, trange: -2.020 .0 ) [25]. The resulting txt file was imported in Python as a Pandas DataFrame. For each subject, only the channel with the highest response amplitude was analyzed. The peak response was identified as the maximal value for $\mathrm{THb}$ and $\mathrm{OHb}$ and the minimum value for $\mathrm{DHb}$. A grand average was taken of the 20 trials of data per stimulating condition and differences between visual stimulation 'on' (reversing checkerboard) and 'off' (blank) were compared. All data were normalized with respect to the blankevoked response using a subtraction method. Statistical analysis was carried out using pingouin Python library [109] and the following functions: pingouin.ttest (paired and two-sided t-test), pingouin.rm_anova (one-way repeated measures ANOVA), pingouin.pairwise_ttests (post hoc analysis), pingouin.pairwise_corr (Spearman correlation), pingouin.regplot (Linear regression). T-test, ANOVA, and post hoc analysis were used to assess differences in fNIRS peak responses following different stimulating 
conditions, whereas we tested the interaction between the amplitude of fNIRS measures and AQ scores with Spearman correlation. Total $A Q$ scores,

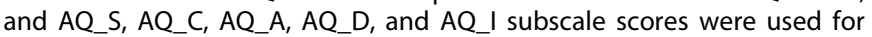
correlational analysis. We employed the Linear regression to plot such correlations. For the correlational analysis adjustments for multiple comparisons were performed using the Benjamini/Hochberg false discovery rate (BH-FDR) correction. The effect size calculated for the ANOVA was the generalized eta-squared. All the plots have been generated using Matplotlib Python library [110]. All statistical metrics and details are reported in Supplementary Table 1 (Table S1).

\section{DATA AVAILABILITY}

The datasets generated during the current study and scripts used for visual stimulation are available, respectively on Zenodo (https://doi.org/10.5281/ zenodo.5101912) and GitHub website (https://github.com/raffaelemazziotti/ FNIRS_code).

\section{REFERENCES}

1. Maenner MJ, Rice CE, Arneson CL, Cunniff C, Schieve LA, Carpenter LA, et al. Potential impact of DSM-5 criteria on autism spectrum disorder prevalence estimates. JAMA Psychiatry. 2014;71:292-300.

2. Wing L. The Continuum of Autistic Characteristics. In: Schopler E, Mesibov GB, (eds.) Diagnosis and Assessment in Autism. Current Issues in Autism. Boston, MA: Springer.

3. Posserud M-B, Lundervold AJ, Gillberg C. Autistic features in a total population of 7-9-year-old children assessed by the ASSQ (Autism Spectrum Screening Questionnaire). J Child Psychol Psychiatry. 2006;47:167-75.

4. Ruzich $E$, Allison $C$, Smith $P$, Watson $P$, Auyeung B, Ring $H$, et al. Measuring autistic traits in the general population: A systematic review of the AutismSpectrum Quotient (AQ) in a nonclinical population sample of 6,900 typical adult males and females. Mol Autism. 2015;6:2.

5. Persico AM, Bourgeron T. Searching for ways out of the autism maze: Genetic, epigenetic and environmental clues. Trends Neurosci. 2006;29:349-58.

6. Ruzich E, Allison C, Smith P, Watson P, Auyeung B, Ring H, et al. Subgrouping siblings of people with autism: Identifying the broader autism phenotype. Autism Res. 2016;9:658-65.

7. Piven J, Palmer P, Jacobi D, Childress D, Arndt S. Broader autism phenotype: Evidence from a family history study of multiple-incidence autism families. Am J Psychiatry. 1997;154:185-90.

8. Billeci L, Calderoni S, Conti E, Gesi C, Carmassi C, Dell'Osso L, et al. The broad autism (Endo)phenotype: Neurostructural and neurofunctional correlates in parents of individuals with autism spectrum disorders. Front Neurosci. 2016;10:346.

9. Carpita B, Carmassi C, Calderoni S, Muti D, Muscarella A, Massimetti G, et al. The broad autism phenotype in real-life: clinical and functional correlates of autism spectrum symptoms and rumination among parents of patients with autism spectrum disorder. CNS Spectr. 2020;25:765-73.

10. Ozonoff S, Young GS, Carter A, Messinger D, Yirmiya N, Zwaigenbaum L, et al. Recurrence risk for autism spectrum disorders: a Baby Siblings Research Consortium study. Pediatrics. 2011;128:e488-95.

11. Messinger D, Young GS, Ozonoff S, Dobkins K, Carter A, Zwaigenbaum L, et al. Beyond autism: A baby siblings research consortium study of high-risk children at three years of age. J Am Acad Child Adolesc Psychiatry. 2013;52:300-.e1.

12. Charman T, Young GS, Brian J, Carter A, Carver LJ, Chawarska K, et al. NonASD outcomes at 36 months in siblings at familial risk for autism spectrum disorder (ASD): A baby siblings research consortium (BSRC) study. Autism Res. 2017;10:169-78.

13. Pagnozzi AM, Conti E, Calderoni S, Fripp J, Rose SE. A systematic review of structural MRI biomarkers in autism spectrum disorder: A machine learning perspective. Int J Dev Neurosci. 2018;71:68-82

14. Frye RE, Vassall $S$, Kaur G, Lewis $C$, Karim M, Rossignol D. Emerging biomarkers in autism spectrum disorder: A systematic review. Ann Transl Med. 2019;7:792.

15. Amaral DG, Schumann CM, Nordahl CW. Neuroanatomy of autism. Trends Neurosci. 2008;31:137-45.

16. Bellani $M$, Calderoni S, Muratori F, Brambilla P. Brain anatomy of autism spectrum disorders I. Focus on corpus callosum. Epidemiol Psychiatr Sci. 2013;22:217-21.

17. Bellani M, Calderoni S, Muratori F, Brambilla P. Brain anatomy of autism spectrum disorders II. Focus on amygdala. Epidemiol Psychiatr Sci. 2013;22:309-12.

18. Billeci L, Sicca F, Maharatna K, Apicella F, Narzisi A, Campatelli G, et al. On the application of quantitative EEG for characterizing autistic brain: A systematic review. Front Hum Neurosci. 2013;7:442.

19. Calderoni S, Bellani M, Hardan AY, Muratori F, Brambilla P. Basal ganglia and restricted and repetitive behaviours in Autism Spectrum Disorders: Current status and future perspectives. Epidemiol Psychiatr Sci. 2014;23:235-8.
20. Bralten J, van Hulzen KJ, Martens MB, Galesloot TE, Arias Vasquez A, Kiemeney LA, et al. Autism spectrum disorders and autistic traits share genetics and biology. Mol Psychiatry. 2018;23:1205-12.

21. Lloyd-Fox S, Blasi A, Elwell CE. Illuminating the developing brain: The past, present, and future of functional near infrared spectroscopy. Neurosci Biobehav Rev. 2010;34:269-84.

22. Gervain J, Mehler J, Werker JF, Nelson CA, Csibra G, Lloyd-Fox S, et al. Nearinfrared spectroscopy: A report from the McDonnell infant methodology consortium. Dev Cogn Neurosci. 2011;1:22-46.

23. Raichle ME, Mintun MA. Brain work and brain imaging. Annu Rev Neurosci. 2006:29:449-76.

24. Vanderwert RE, Nelson CA. The use of near-infrared spectroscopy in the study of typical and atypical development. Neuroimage. 2014;85:264-71. Pt 1

25. Di Lorenzo R, Pirazzoli L, Blasi A, Bulgarelli C, Hakuno Y, Minagawa Y, et al. Recommendations for motion correction of infant fNIRS data applicable to multiple data sets and acquisition systems. Neuroimage. 2019;200:511-27.

26. Yamasaki T, Ogata K, Maekawa T, ljichi I, Katagiri M, Mitsudo T, et al. Rapid maturation of voice and linguistic processing systems in preschool children: $A$ near-infrared spectroscopic study. Exp Neurol. 2013;250:313-20.

27. Mazzoni A, Grove R, Eapen V, Lenroot RK, Bruggemann J. The promise of functional near-infrared spectroscopy in autism research: What do we know and where do we go? Soc Neurosci. 2019;14:505-18.

28. Zhang $F$, Roeyers $H$. Exploring brain functions in autism spectrum disorder: $A$ systematic review on functional near-infrared spectroscopy (fNIRS) studies. Int J Psychophysiol. 2019;137:41-53.

29. Keehn B, Wagner JB, Tager-Flusberg H, Nelson CA. Functional connectivity in the first year of life in infants at-risk for autism: A preliminary near-infrared spectroscopy study. Front Hum Neurosci. 2013;7:444.

30. Zhu H, Fan Y, Guo H, Huang D, He S. Reduced interhemispheric functional connectivity of children with autism spectrum disorder: Evidence from functional near infrared spectroscopy studies. Biomed Opt Express. 2014;5:1262-74.

31. Li J, Qiu L, Xu L, Pedapati EV, Erickson CA, Sunar U. Characterization of autism spectrum disorder with spontaneous hemodynamic activity. Biomed Opt Express. 2016;7:3871-81.

32. Li Y, Yu D. Weak network efficiency in young children with Autism Spectrum Disorder: Evidence from a functional near-infrared spectroscopy study. Brain Cognition. 2016;108:47-55.

33. Jia $\mathrm{H}, \mathrm{Li} Y, \mathrm{Yu}$ D. Attenuation of long-range temporal correlations of neuronal oscillations in young children with autism spectrum disorder. Neuroimage Clin. 2018;20:424-32.

34. Cao W, Zhu H, Li Y, Wang Y, Bai W, Lao $U$ et al. The development of brain network in males with autism spectrum disorders from childhood to adolescence: Evidence from fNIRS study. Brain Sci. 2021;11:120. https://doi.org/ 10.3390/brainsci11010120.

35. Xu M, Minagawa Y, Kumazaki H, Okada K-I, Naoi N. Prefrontal responses to odors in individuals with autism spectrum disorders: Functional NIRS measurement combined with a fragrance pulse ejection system. Front Hum Neurosci. 2020;14:523456.

36. Xiao T, Xiao Z, Ke X, Hong S, Yang H, Su Y, et al. Response inhibition impairment in high functioning autism and attention deficit hyperactivity disorder: Evidence from near-infrared spectroscopy data. PLoS One. 2012;7:e46569.

37. Lloyd-Fox S, Blasi A, Elwell CE, Charman T, Murphy D, Johnson MH. Reduced neural sensitivity to social stimuli in infants at risk for autism. Proc Biol Sci. 2013;280:20123026.

38. Braukmann $\mathrm{R}$, Lloyd-Fox $\mathrm{S}$, Blasi A, Johnson $\mathrm{MH}$, Bekkering $\mathrm{H}$, Buitelaar JK, et al. Diminished socially selective neural processing in 5-month-old infants at high familial risk of autism. Eur J Neurosci. 2018;47:720-8.

39. Lloyd-Fox S, Blasi A, Pasco G, Gliga T, Jones EJH, Murphy DGM, et al. Cortical responses before 6 months of life associate with later autism. Eur J Neurosci. 2018;47:736-49.

40. Bhat AN, McDonald NM, Eilbott JE, Pelphrey KA. Exploring cortical activation and connectivity in infants with and without familial risk for autism during naturalistic social interactions: A preliminary study. Infant Behav Dev. 2019;57:101337.

41. Iwanaga R, Tanaka G, Nakane H, Honda S, Imamura A, Ozawa H. Usefulness of near-infrared spectroscopy to detect brain dysfunction in children with autism spectrum disorder when inferring the mental state of others. Psychiatry Clin Neurosci. 2013;67:203-9.

42. Zhu B, Godavarty A. Functional connectivity in the brain in joint attention skills using near infrared spectroscopy and imaging. Behav Brain Res. 2013;250:28-31.

43. Zhu H, Li J, Fan Y, Li X, Huang D, He S. Atypical prefrontal cortical responses to joint/non-joint attention in children with autism spectrum disorder (ASD): $A$ functional near-infrared spectroscopy study. Biomed Opt Express. 2015;6:690-701.

44. Tamura R, Kitamura $H$, Endo $T$, Abe R, Someya T. Decreased leftward bias of prefrontal activity in autism spectrum disorder revealed by functional nearinfrared spectroscopy. Psychiatry Res. 2012;203:237-40. 
45. Su W-C, Culotta M, Mueller J, Tsuzuki D, Pelphrey K, Bhat A. Differences in cortical activation patterns during action observation, action execution, and interpersonal synchrony between children with or without autism spectrum disorder (ASD): An fNIRS pilot study. PLoS One. 2020;15:e0240301.

46. Kita Y, Gunji A, Inoue Y, Goto T, Sakihara K, Kaga M, et al. Self-face recognition in children with autism spectrum disorders: A near-infrared spectroscopy study. Brain Dev. 2011;33:494-503.

47. Nakadoi Y, Sumitani S, Watanabe Y, Akiyama M, Yamashita N, Ohmori T. Multichannel near-infrared spectroscopy shows reduced activation in the prefrontal cortex during facial expression processing in pervasive developmental disorder. Psychiatry Clin Neurosci. 2012;66:26-33.

48. Fox SE, Wagner JB, Shrock CL, Tager-Flusberg H, Nelson CA. Neural processing of facial identity and emotion in infants at high-risk for autism spectrum disorders. Front Hum Neurosci. 2013;7:89.

49. Mori K, Toda Y, Ito H, Mori T, Mori K, Goji A, et al. Neuroimaging in autism spectrum disorders: 1H-MRS and NIRS study. J Med Invest. 2015;62:29-36.

50. Minagawa-Kawai Y, Naoi N, Kikuchi N, Yamamoto J-I, Nakamura K, Kojima S. Cerebral laterality for phonemic and prosodic cue decoding in children with autism. Neuroreport. 2009;20:1219-24.

51. Funabiki Y, Murai T, Toichi M. Cortical activation during attention to sound in autism spectrum disorders. Res Dev Disabil. 2012;33:518-24.

52. Edwards LA, Wagner JB, Tager-Flusberg H, Nelson CA. Differences in neural correlates of speech perception in 3 month olds at high and low risk for Autism Spectrum Disorder. J Autism Dev Disord. 2017:47:3125-38.

53. Pecukonis $M$, Perdue $K L$, Wong J, Tager-Flusberg $H$, Nelson CA. Exploring the relation between brain response to speech at 6-months and language outcomes at 24-months in infants at high and low risk for autism spectrum disorder: A preliminary functional near-infrared spectroscopy study. Dev Cogn Neurosci. 2021;47:100897.

54. Yasumura A, Omori M, Fukuda A, Takahashi J, Yasumura $Y$, Nakagawa E, et al. Age-related differences in frontal lobe function in children with ADHD. Brain Dev. 2019;41:577-86.

55. Chou $\mathrm{P}-\mathrm{H}$, Huang $\mathrm{C}-\mathrm{J}$, Sun $\mathrm{C}-\mathrm{W}$. The potential role of functional near-infrared spectroscopy as clinical biomarkers in schizophrenia. Curr Pharm Des. 2020;26:201-17.

56. Husain SF, Yu R, Tang T-B, Tam WW, Tran B, Quek TT, et al. Validating a functional near-infrared spectroscopy diagnostic paradigm for Major Depressive Disorder. Sci Rep. 2020;10:9740.

57. Xu L, Liu Y, Yu J, Li X, Yu X, Cheng H, et al. Characterizing autism spectrum disorder by deep learning spontaneous brain activity from functional nearinfrared spectroscopy. J Neurosci Methods. 2020;331:108538.

58. Yang D, Hong K-S, Yoo S-H, Kim C-S. Evaluation of neural degeneration biomarkers in the prefrontal cortex for early identification of patients with mild cognitive impairment: An fNIRS study. Front Hum Neurosci. 2019;13:317.

59. Yanagisawa K, Nakamura N, Tsunashima H, Narita N. Proposal of auxiliary diagnosis index for autism spectrum disorder using near-infrared spectroscopy. Neurophotonics. 2016;3:031413.

60. Xu L, Sun Z, Xie J, Yu J, Li J, Wang J. Identification of autism spectrum disorder based on short-term spontaneous hemodynamic fluctuations using deep learning in a multi-layer neural network. Clin Neurophysiol. 2021;132:457-68.

61. Durand S, Patrizi A, Quast KB, Hachigian L, Pavlyuk R, Saxena A, et al. NMDA receptor regulation prevents regression of visual cortical function in the absence of Mecp2. Neuron. 2012;76:1078-90.

62. de Freitas Dotto P, Cavascan NN, Berezovsky A, Sacai PY, Rocha DM, Pereira JM et al. Sweep visually evoked potentials and visual findings in children with West syndrome. Eur J Paediatr Neurol. 2014;18:201-10.

63. Begenisic T, Sansevero G, Baroncelli L, Cioni G, Sale A. Early environmental therapy rescues brain development in a mouse model of Down syndrome. Neurobiol Dis. 2015;82:409-19.

64. Boggio EM, Pancrazi L, Gennaro M, Lo Rizzo C, Mari F, Meloni I, et al. Visual impairment in FOXG1-mutated individuals and mice. Neuroscience. 2016;324:496-508.

65. Mazziotti R, Lupori L, Sagona G, Gennaro M, Della Sala G, Putignano E, et al. Searching for biomarkers of CDKL5 disorder: early-onset visual impairment in CDKL5 mutant mice. Hum Mol Genet. 2017;26:2290-8.

66. Mazziotti R, Cacciante F, Sagona G, Lupori L, Gennaro M, Putignano E, et al. Novel translational phenotypes and biomarkers for creatine transporter deficiency. Brain Commun. 2020;2:fcaa089.

67. LeBlanc JJ, DeGregorio G, Centofante E, Vogel-Farley VK, Barnes K, Kaufmann WE, et al. Visual evoked potentials detect cortical processing deficits in Rett syndrome. Ann Neurol. 2015;78:775-86.

68. Keehn B, Westerfield M, Townsend J. Brief report: Cross-modal capture: Preliminary evidence of inefficient filtering in children with Aautism Spectrum Disorder. J Autism Dev Disord. 2019:49:385-90.
69. Little J-A. Vision in children with autism spectrum disorder: A critical review. Clin Exp Optom. 2018;101:504-13.

70. Seymour RA, Rippon G, Gooding-Williams G, Schoffelen JM, Kessler K. Dysregulated oscillatory connectivity in the visual system in autism spectrum disorder. Brain. 2019;142:3294-305.

71. Spiegel A, Mentch J, Haskins AJ, Robertson CE. Slower binocular rivalry in the autistic brain. Curr Biol. 2019;29:2948-.e3.

72. Kovarski K, Malvy J, Khanna RK, Arsène S, Batty M, Latinus M. Reduced visual evoked potential amplitude in autism spectrum disorder, a variability effect? Transl Psychiatry. 2019;9:341.

73. Wijeakumar S, Shahani U, Simpson WA, McCulloch DL. Localization of hemodynamic responses to simple visual stimulation: An fNIRS study. Investigative Opthalmology Vis Sci. 2012;53:2266.

74. Chen L-C, Sandmann P, Thorne JD, Herrmann CS, Debener S. Association of concurrent fNIRS and EEG signatures in response to auditory and visual stimuli. Brain Topogr. 2015;28:710-25.

75. Pinti P, Tachtsidis I, Hamilton A, Hirsch J, Aichelburg C, Gilbert S, et al. The present and future use of functional near-infrared spectroscopy (fNIRS) for cognitive neuroscience. Ann N. Y Acad Sci. 2020;1464:5-29.

76. Baron-Cohen S, Wheelwright S, Skinner R, Martin J, Clubley E. The autism-spectrum quotient (AQ): Evidence from Asperger syndrome/high-functioning autism, males and females, scientists and mathematicians. J Autism Dev Disord. 2001;31:5-17.

77. Auyeung B, Baron-Cohen S, Wheelwright S, Allison C. The Autism Spectrum Quotient: Children's version (AQ-Child). J Autism Dev Disord. 2008;38:1230-40.

78. Tachtsidis I, Scholkmann F. False positives and false negatives in functional nearinfrared spectroscopy: Issues, challenges, and the way forward. Neurophotonics. 2016;3:031405.

79. Ward LM, Aitchison RT, Tawse M, Simmers AJ, Shahani U. Reduced haemodynamic response in the ageing visual cortex measured by absolute fNIRS. PLoS One. 2015;10:e0125012.

80. Simmons DR, Robertson AE, McKay LS, Toal E, McAleer P, Pollick FE. Vision in autism spectrum disorders. Vis Res. 2009;49:2705-39.

81. Park WJ, Schauder KB, Zhang R, Bennetto L, Tadin D. High internal noise and poor external noise filtering characterize perception in autism spectrum disorder. Sci Rep. 2017;7:17584.

82. Noel J-P, Lakshminarasimhan KJ, Park H, Angelaki DE. Increased variability but intact integration during visual navigation in Autism Spectrum Disorder. Proc Natl Acad Sci USA. 2020;117:11158-66.

83. Noel J-P, Zhang L-Q, Stocker AA, Angelaki DE. Individuals with autism spectrum disorder have altered visual encoding capacity. PLoS Biol. 2021;19:e3001215.

84. Turi $M$, Burr DC, Binda P. Pupillometry reveals perceptual differences that are tightly linked to autistic traits in typical adults. Elife 2018;7:e32399. https://doi. org/10.7554/eLife.32399.

85. Tortelli C, Turi M, Burr DC, Binda P. Objective pupillometry shows that perceptual styles covary with autistic-like personality traits. Elife 2021;10:e67185. https://doi.org/10.7554/eLife.67185.

86. Van der Hallen R, Evers K, Brewaeys K, Van den Noortgate W, Wagemans J. Global processing takes time: A meta-analysis on local-global visual processing in ASD. Psychological Bull. 2015;141:549-73.

87. Ouellette J, Toussay X, Comin CH, Costa L, da F, Ho M, et al. Vascular contributions to $16 \mathrm{p} 11.2$ deletion autism syndrome modeled in mice. Nat Neurosci. 2020;23:1090-101.

88. Kozberg M, Hillman E. Neurovascular coupling and energy metabolism in the developing brain. Prog Brain Res. 2016;225:213-42.

89. Harris JJ, Reynell C, Attwell D. The physiology of developmental changes in BOLD functional imaging signals. Dev Cogn Neurosci. 2011;1:199-216.

90. Broadbent J, Galic I, Stokes MA. Validation of Autism Spectrum Quotient adult version in an Australian sample. Autism Res Treat. 2013;2013:1-7.

91. Lundqvist L-O, Lindner $\mathrm{H}$. Is the Autism-Spectrum Quotient a valid measure of traits associated with the autism spectrum? A Rasch validation in adults with and without autism spectrum disorders. J Autism Dev Disord. 2017;47:2080-91.

92. Wouters SGM, Spek AA. The use of the Autism-spectrum Quotient in differentiating high-functioning adults with autism, adults with schizophrenia, and a neurotypical adult control group. Res Autism Spectr Disord. 2011;5:1169-75

93. Leekam SR, Nieto C, Libby SJ, Wing L, Gould J. Describing the sensory abnormalities of children and adults with autism. J Autism Dev Disord. 2007;37:894-910.

94. Scharre JE, Creedon MP. Assessment of visual function in autistic children Optom Vis Sci. 1992;69:433-9.

95. Apicella F, Costanzo V, Purpura G. Are early visual behavior impairments involved in the onset of autism spectrum disorders? Insights for early diagnosis and intervention. Eur J Pediatrics. 2020;179:225-34.

96. Kern JK, Trivedi MH, Grannemann BD, Garver CR, Johnson DG, Andrews AA, et al Sensory correlations in autism. Autism 2007:11:123-34. 
97. Jones W, Klin A. Attention to eyes is present but in decline in 2-6-month-old infants later diagnosed with autism. Nature. 2013;504:427-31.

98. McPartland JC, Lerner MD, Bhat A, Clarkson T, Jack A, Koohsari S et al. Looking back at the next 40 years of ASD neuroscience research. J Autism Dev Disord. 2021. https://doi.org/10.1007/s10803-021-05095-5.

99. Baird G, Cass H, Slonims V. Diagnosis of autism. BMJ. 2003;327:488-93.

100. Zwaigenbaum L, Bryson SE, Brian J, Smith IM, Roberts W, Szatmari P, et al. Stability of diagnostic assessment for autism spectrum disorder between 18 and 36 months in a high-risk cohort. Autism Res. 2016;9:790-800.

101. Emerson RW, Adams C, Nishino T, Hazlett HC, Wolff JJ, Zwaigenbaum L et al. Functional neuroimaging of high-risk 6-month-old infants predicts a diagnosis of autism at 24 months of age. Sci Transl Med. 2017. https://doi.org/ 10.1126/scitranslmed.aag2882.

102. Hazlett HC, Gu H, Munsell BC, Kim SH, Styner M, Wolff J, et al. Early brain development in infants at high risk for autism spectrum disorder. Nature. 2017;542:348-51.

103. Stokes MA, Kornienko L, Scheeren AM, Koot HM, Begeer S. A comparison of children and adolescent's self-report and parental report of the PedsQL among those with and without autism spectrum disorder. Qual Life Res. 2017;26:611-24.

104. Ruta L, Mazzone D, Mazzone L, Wheelwright S, Baron-Cohen S. The AutismSpectrum Quotient-Italian version: A cross-cultural confirmation of the broader autism phenotype. J Autism Dev Disord. 2012;42:625-33.

105. Vrana A, Meier ML, Hotz-Boendermaker S, Humphreys BK, Scholkmann F. Cortical sensorimotor processing of painful pressure in patients with chronic lower Back pain —an optical neuroimaging study using fNIRS. Front Hum Neurosci. 2016;10:578.

106. Zimeo Morais GA, Balardin JB, Sato JR. fNIRS Optodes' Location Decider (fOLD): A toolbox for probe arrangement guided by brain regions-of-interest. Sci Rep. 2018;8:3341.

107. Peirce J, Gray JR, Simpson S, MacAskill M, Höchenberger R, Sogo H, et al. PsychoPy2: Experiments in behavior made easy. Behav Res Methods. 2019;51:195-203.

108. Gollapudi S. OpenCV with Python. In Learn Computer Vision Using OpenCV. 2019. p. 31-50.

109. Vallat R. Pingouin: Statistics in Python. J Open Source Softw. 2018;3:1026.

110. Hunter JD. Matplotlib: A 2D graphics environment. Comput Sci Eng. 2007;9:90-95.

\section{ACKNOWLEDGEMENTS}

This work was supported by a Telethon grant from GP19177 to LB, a grant from University of Pisa (PRA-2020-50) to RB, and Italian Ministry of Health, RC 2021. EC also acknowledges AIMS-2-Trials europea project.

\section{AUTHOR CONTRIBUTIONS}

$R B, G C$, and $L B$ conceived the study. RM, ES, EC, VM, RR, and LB designed the experiments. RM, ES, and $L B$ carried out the research. RM, ES, and $L B$ analyzed the data. LB wrote the manuscript. All authors were involved in the revision of the draft manuscript and have agreed to the final content.

\section{CONFLICT OF INTEREST}

The authors declare no conflict of interest.

\section{ADDITIONAL INFORMATION}

Supplementary information The online version contains supplementary material available at https://doi.org/10.1038/s41398-022-01820-5.

Correspondence and requests for materials should be addressed to Laura Baroncelli.

Reprints and permission information is available at http://www.nature.com/ reprints

Publisher's note Springer Nature remains neutral with regard to jurisdictional claims in published maps and institutional affiliations.

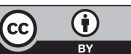

Open Access This article is licensed under a Creative Commons Attribution 4.0 International License, which permits use, sharing, adaptation, distribution and reproduction in any medium or format, as long as you give appropriate credit to the original author(s) and the source, provide a link to the Creative Commons license, and indicate if changes were made. The images or other third party material in this article are included in the article's Creative Commons license, unless indicated otherwise in a credit line to the material. If material is not included in the article's Creative Commons license and your intended use is not permitted by statutory regulation or exceeds the permitted use, you will need to obtain permission directly from the copyright holder. To view a copy of this license, visit http://creativecommons. org/licenses/by/4.0/.

(c) The Author(s) 2022 